\title{
A Rare Opportunist, Morganella morganii, Decreases Severity of Polymicrobial Catheter-Associated Urinary Tract Infection
}

\author{
Brian S. Learman, ${ }^{a}$ Aimee L. Brauer, ${ }^{a}$ Kathryn A. Eaton, ${ }^{b}$ (D) Chelsie E. Armbrustera \\ aDepartment of Microbiology and Immunology, Jacobs School of Medicine and Biomedical Sciences, State University of New York at Buffalo, Buffalo, New York, USA \\ baboratory Animal Medicine Unit, University of Michigan Medical School, Ann Arbor, Michigan, USA
}

ABSTRACT Catheter-associated urinary tract infections (CAUTIs) are common hospital-acquired infections and frequently polymicrobial, which complicates effective treatment. However, few studies experimentally address the consequences of polymicrobial interactions within the urinary tract, and the clinical significance of polymicrobial bacteriuria is not fully understood. Proteus mirabilis is one of the most common causes of monomicrobial and polymicrobial CAUTI and frequently cocolonizes with Enterococcus faecalis, Escherichia coli, Providencia stuartii, and Morganella morganii. P. mirabilis infections are particularly challenging due to its potent urease enzyme, which facilitates formation of struvite crystals, catheter encrustation, blockage, and formation of urinary stones. We previously determined that interactions between $P$. mirabilis and other uropathogens can enhance $P$. mirabilis urease activity, resulting in greater disease severity during experimental polymicrobial infection. Our present work reveals that $M$. morganii acts on $P$. mirabilis in a contact-independent manner to decrease urease activity. Furthermore, $M$. morganii actively prevents urease enhancement by $E$. faecalis, $P$. stuartii, and $E$. coli. Importantly, these interactions translate to modulation of disease severity during experimental CAUTI, predominantly through a urease-dependent mechanism. Thus, products secreted by multiple bacterial species in the milieu of the catheterized urinary tract can directly impact prognosis.

KEYWORDS UTI, pyelonephritis, bacterial infections, medical devices, catheter, CAUTI, Proteus mirabilis, bacteremia, urolithiasis, urease, Enterococcus faecalis, Escherichia coli, Morganella morganii, urinary tract infection, polymicrobial

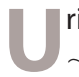
rinary catheters are common in health care settings, estimated to be utilized in $\sim 60 \%$ of critically ill patients, $20 \%$ of surgical unit patients, and $10 \%$ of nursing homes residents (1-3). The vast majority of individuals with an indwelling urinary catheter will experience bacteriuria and may progress to catheter-associated urinary tract infection (CAUTI) (1). CAUTI is the most common health care-associated condition in the United States and carries an estimated economic burden approaching \$1.7 billion annually (4).

Bacteriuria in catheterized individuals rapidly becomes polymicrobial, involving combinations of Proteus mirabilis, Enterococcus faecalis, Escherichia coli, Pseudomonas aeruginosa, Klebsiella pneumoniae, Providencia stuartii, Morganella morganii, Citrobacter species, Staphylococcus species, and Streptococcus species $(1,2,5)$. It is estimated that 31 to $86 \%$ of CAUTIs are also polymicrobial (6-14); however, urine specimens are often suspected of harboring periurethral or vaginal microbiota if multiple colony types are observed, particularly if Gram-positive organisms are present, and are therefore often dismissed as "contamination" $(15,16)$. Thus, the clinical significance of polymicrobial bacteriuria is not fully understood.

There are numerous experimental and clinical examples of polymicrobial coloniza-
Citation Learman BS, Brauer AL, Eaton KA Armbruster CE. 2020. A rare opportunist, Morganella morganii, decreases severity of polymicrobial catheter-associated urinary tract infection. Infect Immun 88:e00691-19. https:// doi.org/10.1128/IAl.00691-19.

Editor Marvin Whiteley, Georgia Institute of Technology School of Biological Sciences Copyright $\odot 2019$ American Society for Microbiology. All Rights Reserved.

Address correspondence to Chelsie E. Armbruster, chelsiea@buffalo.edu.

Received 30 August 2019

Returned for modification 30 September 2019

Accepted 4 October 2019

Accepted manuscript posted online 14

October 2019

Published 17 December 2019 
tion causing more severe disease than infection with a single bacterial species (monomicrobial), including periodontitis, abdominal abscesses, actinomycosis, chronic wounds, otitis media, pneumonia, cystic fibrosis, inflammatory bowel disease, uncomplicated urinary tract infection, and CAUTI (17-20). In many cases, polymicrobial interactions promote an increased level of colonization for one or both of the bacterial species. It is also possible for a species that is generally perceived as a commensal organism to become an "accessory pathogen" by supporting or enhancing the virulence of a more traditional pathogen (21). In contrast to these disease-promoting interactions, other polymicrobial interactions attenuate disease severity or exclude colonization by traditional pathogens (21). Considering the prevalence of polymicrobial bacteriuria and CAUTI in catheterized individuals, exploration of polymicrobial interactions and their impact on disease severity is necessary.

Our recent analysis of 182 clinical CAUTIs from a 3-year study at 12 nursing homes identified $P$. mirabilis as the most common cause of CAUTI (26\% of cases), followed by Enterococcus species (21\%) and Escherichia coli (20\%) (22). Of the 182 CAUTIs, 31\% were polymicrobial, and the most common bacterial combination was $P$. mirabilis with $E$. faecalis, which is in agreement with prior work regarding the composition of polymicrobial CAUTI (1, 5, 12-14, 22, 23). Notably, E. faecalis, M. morganii, and $P$. stuartii were significantly more prevalent during polymicrobial CAUTI than monomicrobial infection, and each of these bacterial species has been reported to cocolonize catheters with $P$. mirabilis $(1,5,12-14,22,24)$. These five species therefore represent important constituents of the polymicrobial CAUTI environment.

P. mirabilis poses a significant challenge for effective CAUTI treatment as Proteus isolates are intrinsically drug resistant, exhibiting high tolerance to tetracycline and polymyxin, and clinical isolates are often resistant to aminoglycosides and fluoroquinolones (25). Furthermore, there are increasing reports of isolates producing extendedspectrum $\beta$-lactamases and carbapenemases (25-29), which threatens the utility of last-resort antibiotics and increases the mortality rate for $P$. mirabilis infection (30-32). $P$. mirabilis also acts as a "hub" species in catheterized nursing home residents, promoting colonization by additional multidrug resistance organisms (33) and providing protection from antibiotic treatment (34). Furthermore, $P$. mirabilis produces a potent urease enzyme that hydrolyzes the urea in urine to carbon dioxide and ammonia, thereby increasing urine $\mathrm{pH}$ and facilitating the precipitation of polyvalent ions and resulting in struvite crystals, catheter encrustation, blockage, and formation of urinary stones (urolithiasis) (35-37). Urolithiasis in humans and animal models of infection can elicit bladder obstruction and renal damage $(35,38,39)$, which facilitate sepsis and bacteremia. Indeed, P. mirabilis is the causative agent in 13 to $21 \%$ of bacteremias experienced by nursing home residents, the majority of which are secondary to CAUTI $(9,40-45)$. However, there are examples of catheterized patients with prolonged colonization by $P$. mirabilis who do not experience catheter blockage or urolithiasis $(12,46)$, indicating that the magnitude of struvite crystal formation is likely affected by other factors within the urinary tract environment.

In our prior investigations of $P$. mirabilis urolithiasis and pathogenicity, we determined that numerous common urinary tract colonizers are capable of enhancing $P$. mirabilis urease activity during coculture in urine in vitro (47). E. faecalis, E. coli, and $P$. stuartii were the most potent enhancers of $P$. mirabilis urease activity, and experimental coinfection of $P$. mirabilis with $P$. stuartii in a murine model of complicated UTI resulted in higher urinary $\mathrm{pH}$, a greater incidence of urinary stones, and increased disease severity, all of which were dependent on the presence of a functional urease operon in P. mirabilis $(47,48)$. Thus, the concomitant presence of certain urease-enhancing organisms may correspond to likelihood of developing struvite crystals and urolithiasis during $P$. mirabilis CAUTI. In contrast, $M$. morganii was the only species tested that did not enhance $P$. mirabilis urease in vitro, and may have even dampened activity (47), suggesting that this species may be able to decrease risk of struvite formation during polymicrobial colonization and therefore possibly reduce disease severity.

Like $P$. mirabilis, M. morganii is a urease-positive organism. However, it produces a 
urease enzyme that is distinct from that of $P$. mirabilis (49), and the urine $\mathrm{pH}$ change mediated by $M$. morganii urease activity rarely results in development of struvite crystals or catheter blockage $(24,50-52)$. Interestingly, M. morganii is more commonly isolated from unobstructed catheters than blocked catheters in patients catheterized long-term (12), and precolonization of a catheter by $M$. morganii can actually reduce blockage and encrustation by $P$. mirabilis in vitro (24), albeit transiently. Taken together, these data support the hypothesis that M. morganii may be able to antagonize $P$. mirabilis urease activity and reduce the incidence of struvite crystal formation and urolithiasis during coculture.

In this study, we tested the urease-modulatory capability of $M$. morganii and its impact on disease severity during dual-species and triple-species combinations. We show that $M$. morganii secretes products that decrease $P$. mirabilis urease activity in a contact-independent manner and that the urease-dampening capability of these secreted products can prevent urease enhancement by E. faecalis, P. stuartii, and E. coli. Using a murine model of polymicrobial CAUTI, we further demonstrate that coinfection of $P$. mirabilis with $E$. faecalis dramatically increases disease severity, predominantly due to enhancement of $P$. mirabilis urease activity, and that the addition of $M$. morganii to this polymicrobial combination mitigates disease severity. M. morganii was also capable of mitigating cytotoxicity in vitro. Thus, identification of the M. morganii secreted products that reduce $P$. mirabilis pathogenicity may uncover a novel therapeutic strategy to prevent catheter encrustation, urolithiasis, tissue damage, and secondary bacteremia resulting from $P$. mirabilis catheter colonization.

\section{RESULTS}

Uropathogens exhibit comparable competitive fitness during coculture in dilute human urine. To determine whether $P$. mirabilis exhibits cooperative or competitive behavior with E. faecalis, E. coli, or M. morganii, each bacterial species was cultured independently or cocultured in dilute human urine and bacterial viability was assessed by differential plating at hourly time points until the cultures achieved stationary phase (Fig. 1). The viability of $P$. mirabilis was not significantly impacted by coculture with any of the other species (Fig. 1A), and similar results were observed for E. faecalis (Fig. 1B) and $M$. morganii (Fig. 1C), indicating that these species do not exhibit competitive behavior during coculture in vitro. In contrast, E. coli viability was unaffected by $M$. morganii but dramatically reduced during coculture with $P$. mirabilis (Fig. 1D). The reduction in viability appears to be due to urease-dependent production of ammonia, since $E$. coli viability did not decrease during coculture with a $P$. mirabilis urease mutant ( $\Delta$ ure). A similar urease-dependent impact on viability was previously observed for $P$. stuartii during coculture with $P$. mirabilis (48). Thus, E. coli is sensitive to the increase in urine $\mathrm{pH}$ that occurs due to $P$. mirabilis urease activity, but these species do not appear to exhibit any other competition in vitro.

M. morganii prevents enhancement of $\boldsymbol{P}$. mirabilis urease activity by other bacterial species in a contact-independent manner. We previously demonstrated that numerous uropathogens, including E. faecalis, P. stuartii, and E. coli, can enhance $P$. mirabilis urease activity during coculture in urine while $M$. morganii appears to dampen activity (47). We therefore sought to determine whether urease modulation by these species requires direct cell-cell contact or whether $P$. mirabilis may be responding to factors present in cell-free supernatants. An example of the experimental approach is shown in Fig. 2A. Briefly, each bacterial species of interest was incubated in filtersterilized human urine for $90 \mathrm{~min}$, centrifuged to pellet, and filter sterilized. Cell-free supernatants were also generated from cocultures of $M$. morganii with each of the other bacterial species for comparison to 50:50 mixtures of the monomicrobial culture supernatants. In addition, cell-free supernatant was generated from an isogenic urease mutant of $P$. mirabilis HI4320 ( $\Delta$ ure) for use as a control for the potential impact of nutrient and cofactor depletion on total urease activity. Wild-type $P$. mirabilis HI4320 was then incubated in each of the cell-free supernatants, and the urease activity was measured over a 120-min time course via a colorimetric assay (Fig. 2B and C). The 
A
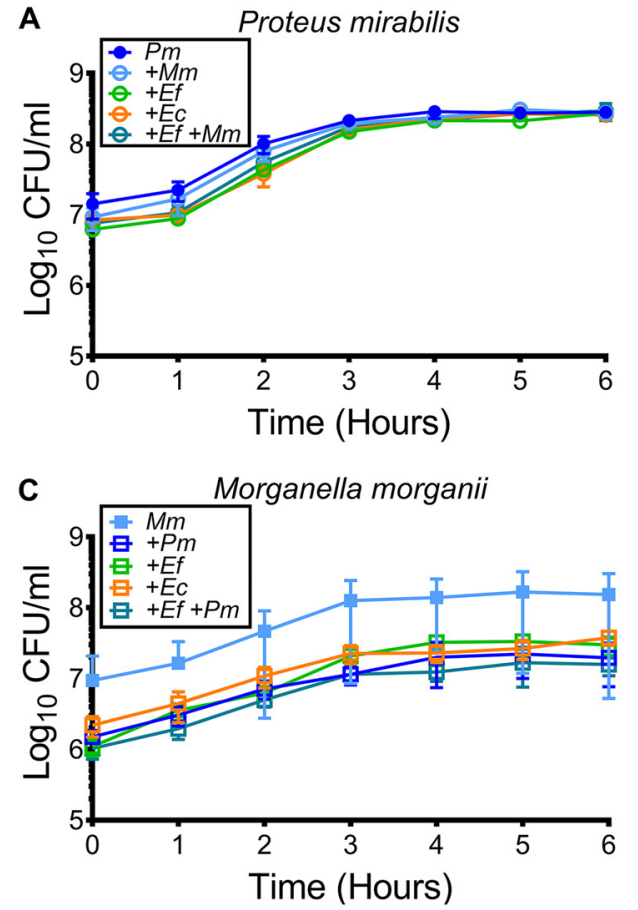

B

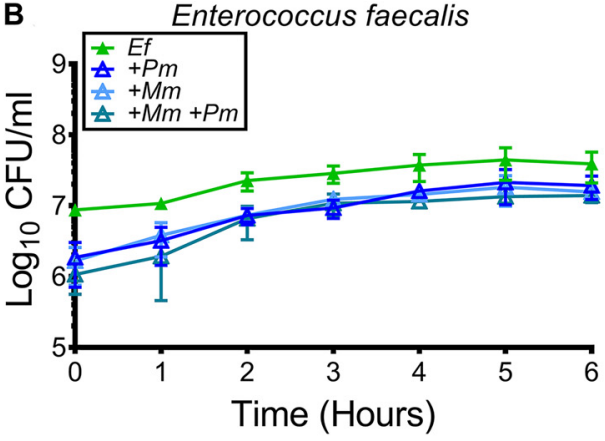

D

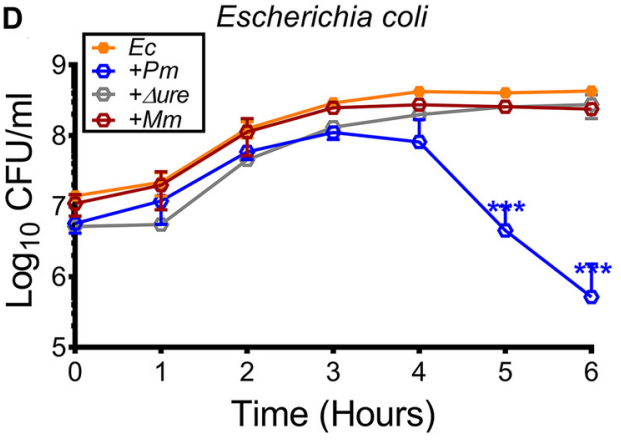

FIG 1 Growth of urinary tract isolates in human urine. P. mirabilis HI4320 (Pm), E. faecalis 3143 (Ef), M. morganii TA43 $(\mathrm{Mm})$, and E. coli CFT073 $(E C)$ were cultured in rich laboratory media overnight, diluted 1:100 into filter-sterilized human urine, and incubated at $37^{\circ} \mathrm{C}$ with aeration for $6 \mathrm{~h}$ for hourly assessment of growth and viability by determination of CFU. (A) CFU of $P$. mirabilis from monomicrobial and polymicrobial cultures. (B) CFU of E. faecalis from monomicrobial and polymicrobial cultures. (C) CFU of M. morganii from monomicrobial and polymicrobial cultures. (D) CFU of E. coli from monomicrobial and polymicrobial cultures, including during incubation with $P$. mirabilis $\Delta$ ure. Error bars represent means \pm the standard deviations (SD) for three independent experiments. Data were $\log _{10}$ transformed for statistical analyses, and significance was assessed by two-way ANOVA with a Dunnett's multiple-comparison test relative to the monomicrobial cultures. ${ }^{* * *}, P<0.001$.

urease activity was also quantified as the mean change in optical density (OD) per minute for the linear portion of the activity curve (Fig. 2D).

$P$. mirabilis urease activity was significantly increased during incubation in cell-free spent urine supernatants from E. faecalis, P. stuartii, and E. coli compared to the level of activity for incubation in supernatant from the isogenic $\Delta$ ure mutant, and the activity was significantly reduced during incubation in supernatant from $M$. morganii (Fig. $2 \mathrm{~B}$ and $D$ ). Therefore, modulation of $P$. mirabilis urease activity by each of these species is mediated by a soluble factor present in spent media and independent of direct cell-cell contact. To determine whether either urease-modulatory activity is dominant, we next assessed urease activity in supernatants generated from cocultures of each species with M. morganii compared to 50:50 mixtures of the monomicrobial supernatants. The urease activity curves for $E$. faecalis and M. morganii are shown in Fig. $2 \mathrm{C}$, and the other coculture urease activity curves are shown in Fig. S1 in the supplemental material. Strikingly, all of the 50:50 mixtures resulted in an intermediate level of urease activity, while all of the coculture supernatants dampened urease activity to a similar level as observed for the M. morganii monomicrobial supernatant (Fig. $2 \mathrm{C}$ and D). Taken together, these results indicate either that $M$. morganii is able to prevent production of the enhancing signal by each of the other bacterial species or that it inactivates, sequesters, or depletes the enhancing signal from the culture supernatant. In either case, $M$. morganii actively prevents enhancement of $P$. mirabilis urease activity by other bacterial species, in addition to directly dampening $P$. mirabilis urease activity.

$M$. morganii prevents enhancement of $\boldsymbol{P}$. mirabilis cytotoxicity by other bacterial species. Our prior studies with $P$. mirabilis and $P$. stuartii demonstrated that coculture increased cytotoxicity to HEK293 cells in vitro compared to monomicrobial cultures and that this process was not dependent upon urease activity from either species (47). We therefore 
A

Incubate in human urine

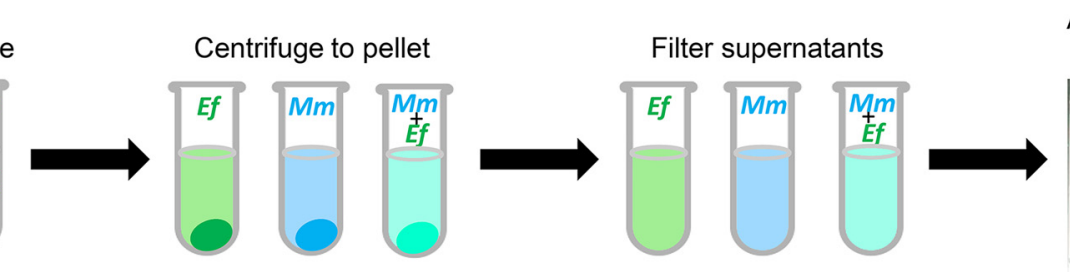

Assess Pm urease activity
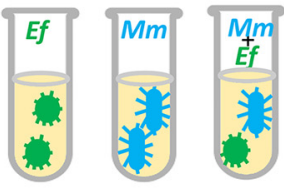

D
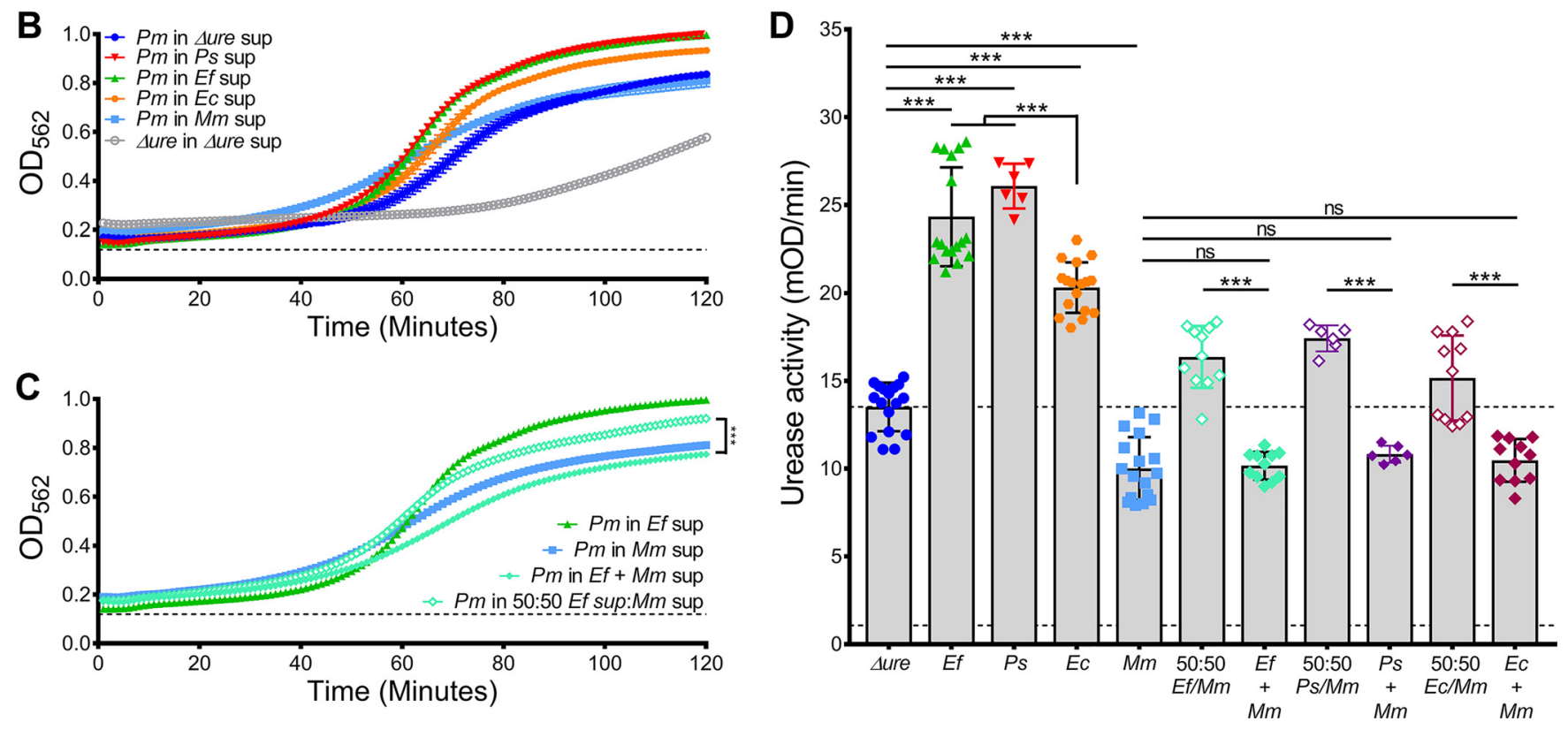

FIG 2 Urease activity of $P$. mirabilis during incubation in cell-free urine supernatants. (A) An example of the experimental approach is displayed. Cell-free urine supernatants were generated from monomicrobial cultures of strains of interest (P. mirabilis $\Delta$ ure, E. faecalis 3143 , E. coli CFT073, and M. morganii TA43) by 90-min incubation in filter-sterilized human urine. Supernatants were also generated from cocultures of $M$. morganii with each of the other bacterial species. P. mirabilis HI4320 was then incubated in each of the cell-free urine supernatants supplemented with excess urea, and urease activity was monitored at 60-s intervals over $120 \mathrm{~min}$. (B) P. mirabilis urease activity in each of the monomicrobial supernatants (sup). (C) P. mirabilis urease activity during incubation in $E$. faecalis and $M$. morganii coculture supernatants compared to a 50:50 mixture of monomicrobial supernatants. Dashed lines indicate the $\mathrm{OD}_{562}$ values of wells containing urine and urea in the absence of bacteria to control for $\mathrm{pH}$ change due to urea breakdown; error bars represent means \pm the SD from a representative experiment with three to six replicates. (D) Urease activity is expressed as the mean change in OD per minute $(\mathrm{mOD} / \mathrm{min}$ ) for the linear portion of the urease activity curves. The dashed lines indicate the average urease activity for $P$. mirabilis during incubation in $P$. mirabilis $\Delta$ ure supernatant and the background level of $\mathrm{pH}$ change due to urea breakdown in the negative-control wells. Error bars represent means \pm the SD from three independent experiments, with at least three replicates each. ns, not significant $(P>0.05) ;{ }^{* * *}, P<0.001$ (as determined by Student $t$ test).

utilized the in vitro cytotoxicity assay to determine whether other bacterial species are capable of enhancing $P$. mirabilis cytotoxicity in a urease-independent manner and whether M. morganii can prevent enhancement. An example of the experimental approach is shown in Fig. 3A. Briefly, each bacterial species of interest was incubated in filter-sterilized human urine for 90 min, washed twice, and overlaid onto a monolayer of HEK293 cells. After a 4-h incubation, cytotoxicity was assessed by measuring the lactate dehydrogenase (LDH) activity in the cell supernatants. For each polymicrobial condition, a predicted level of cytotoxicity $(P)$ was calculated based on the proportion of the bacterial species in the culture and their relative cytotoxicity during monomicrobial culture. The level of cytotoxicity was then expressed relative to that of wild-type P. mirabilis, for ease of comparison between conditions.

Cytotoxicity was first compared for wild-type $P$. mirabilis, the isogenic urease mutant (P. mirabilis $\Delta$ ure), and M. morganii (Fig. 3B). As expected, incubation with the urease mutant resulted in a similar level of cytotoxicity to HEK293 cells as wild-type P. mirabilis, confirming that urease does not contribute to cytotoxicity under these conditions. The urease mutant was therefore utilized for all further cytotoxicity experiments. Interestingly, M. morganii monomicrobial cultures exhibited a level of cytotoxicity similar to 

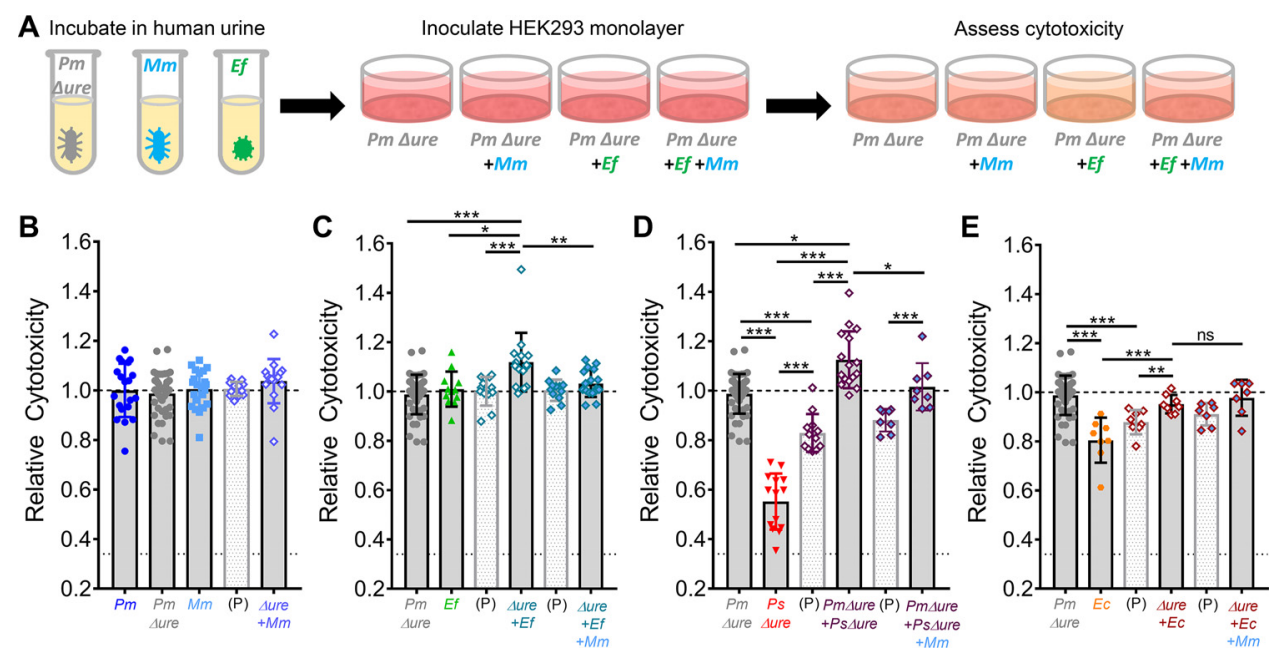

FIG 3 Urease-independent cytotoxicity of monomicrobial and polymicrobial cultures. Cytotoxicity to HEK293 cells was assessed by $\mathrm{LDH}$ release during a 4 -h incubation with various single-species, dual-species, and triple-species combinations as indicated. (A) An example of the experimental approach is displayed. Bacteria were cultured in rich laboratory media to mid-log phase, centrifuged to pellet, resuspended in filter-sterilized human urine, and incubated for 90 min at $37^{\circ} \mathrm{C}$ with aeration prior to infection of HEK293 cells. (B to E) The level of HEK293 lysis under each treatment condition is expressed relative to the level of treatment with $P$. mirabilis $\Delta$ ure (dashed line), and the dotted line indicates background cytotoxicity during treatment with sterile urine. For each dual-species and triple-species combination, a predicted $(P)$ level of cytotoxicity was calculated based on the proportion of each bacterial species in the mixture and the cytotoxicity of their respective monomicrobial cultures. For each panel, the predicted value refers to the same combination of organisms present in the coculture bar immediately to the right. (B) Relative cytotoxicity of $P$. mirabilis HI4320, P. mirabilis $\Delta$ ure, M. morganii, and a dual-species culture of $P$. mirabilis $\Delta$ ure with M. morganii. (C) Relative cytotoxicity of $P$. mirabilis $\Delta$ ure, $E$. faecalis, a dual-species culture of $P$. mirabilis $\Delta$ ure with $E$. faecalis, and a triple-species culture of $P$. mirabilis $\Delta$ ure with $E$. faecalis and M. morganii. (D) Relative cytotoxicity of $P$. mirabilis $\Delta$ ure, $P$. stuartii $\Delta u r e$, a dual-species culture of $P$. mirabilis $\Delta$ ure with $P$. stuartii $\Delta$ ure, and a triple-species culture of $P$. mirabilis $\Delta$ ure with $P$. stuartii $\Delta$ ure and M. morganii. (E) Relative cytotoxicity of $P$. mirabilis $\Delta$ ure, E. coli, a dual-species culture of $P$. mirabilis $\Delta u r e$ with $E$. coli, and a triple-species culture of $P$. mirabilis $\Delta$ ure with $E$. coli and M. morganii. Statistical significance was assessed by nonparametric Mann-Whitney test for indicated comparisons. ns, not significant $(P>0.05) ;{ }^{*}, P<0.05 ;{ }^{* *}$, $P<0.01 ;{ }^{* * *}, P<0.001$.

that of $P$. mirabilis $\Delta$ ure, and coculture had no detectable impact on total cytotoxicity (Fig. 3B).

E. faecalis monomicrobial cultures also exhibited comparable cytotoxicity as $P$. mirabilis $\Delta$ ure, but coculture of $P$. mirabilis $\Delta$ ure with $E$. faecalis significantly increased cytotoxicity over the level observed for the monomicrobial cultures, as well as the predicted value for the dual-species culture (Fig. 3C). Thus, the interaction between these species appears to enhance production of other virulence factors in addition to increasing urease activity. However, the addition of $M$. morganii dampened cytotoxicity back to a level similar to that observed for each respective monomicrobial culture (Fig. 3C). Taken together, these results indicate that coculture of $P$. mirabilis with $E$. faecalis increases cytotoxicity in a urease-independent manner, and $M$. morganii dampens this enhancement.

Similarly, coculture of $P$. mirabilis $\Delta$ ure with a $P$. stuartii urease mutant $(P$. stuartii $\Delta u r e)$ increased cytotoxicity above the level observed for either monomicrobial culture, as well as the predicted coculture level, which is consistent with our previous work (47). For this coculture condition, $M$. morganii was capable of dampening enhancement back down to the level observed for $P$. mirabilis $\Delta$ ure alone, but not quite to the predicted level of activity (Fig. 3D). In contrast, coculture of $P$. mirabilis $\Delta$ ure with $E$. coli resulted in an intermediate level of cytotoxicity that was significantly greater than that $E$. coli alone, as well as the predicted value, but not greater than that of $P$. mirabilis $\Delta$ ure alone (Fig. 3E). However, the addition of M. morganii had no discernible impact on cytotoxicity for this combination (Fig. 3E). In summary, coculture of $P$. mirabilis with $E$. faecalis, $P$. stuartii, or E. coli increases cytotoxicity to various degrees through a ureaseindependent mechanism, and $M$. morganii is capable of preventing increased cytotoxicity from $E$. faecalis and $P$. stuartii, but not $E$. coli. 


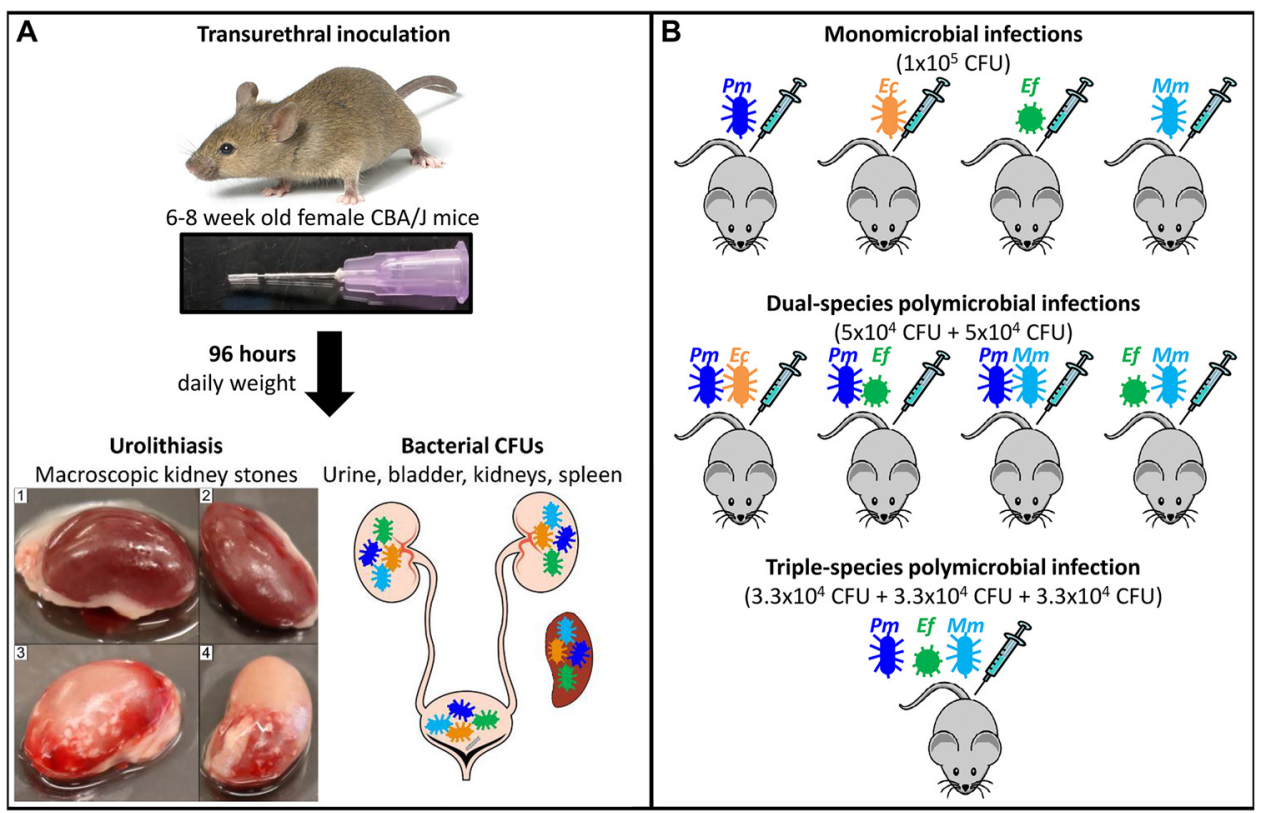

FIG 4 Experimental schematic for murine CAUTI. (A) Overview of experimental approach for murine CAUTI. Female CBA/J mice were purchased from Jackson Laboratories at 5 to 7 weeks of age, allowed to acclimate to the vivarium for 1 week, and infected at 6 to 8 weeks of age by transurethral instillation of $50 \mu$ of a bacterial inoculum over $25 \mathrm{~s}$. At the time of inoculation, a 4-mm segment of sterile silicone catheter tubing was inserted into the bladder. All mice were weighed daily in the morning to monitor weight loss. After $96 \mathrm{~h}$, urine was collected from all mice for determination of bacterial burden, mice were euthanized, and urolithiasis was assessed by the presence of macroscopic kidney stones. Images 1 and 2 display infected kidneys lacking stones, while images 3 and 4 demonstrate the presence of severe macroscopic kidney stones. Bladders, kidneys, and spleens were also removed for determination of bacterial burden. (B) Overview of bacterial inoculations to establish monomicrobial and polymicrobial infections. All mice were inoculated with $1 \times 10^{5}$ CFU of bacteria: Proteus mirabilis HI4320 (Pm, blue), Escherichia coli CFT073 (Ec, orange), Enterococcus faecalis 3143 (Ef, green), and Morganella morganii TA43 (Mm, light blue).

M. morganii dampens disease severity during polymicrobial CAUTI. Our prior studies demonstrated that coinfection of $P$. mirabilis and $P$. stuartii increased disease severity compared to monomicrobial infection in a murine model of UTI and CAUTI. Specifically, enhancement of $P$. mirabilis urease activity by $P$. stuartii resulted in a greater incidence of urolithiasis and bacteremia $(47,48)$. We therefore hypothesized that coinfection of $P$. mirabilis with $E$. faecalis and $E$. coli may similarly increase disease severity and that $M$. morganii may be capable of preventing progression to severe disease during polymicrobial infection.

To test this hypothesis, we utilized our robust animal model of CAUTI via the experimental approached detailed in Fig. 4. Briefly, female CBA/J mice were transurethrally inoculated with $10^{5} \mathrm{CFU}$ of each species alone or dual-species combinations, as follows: (i) P. mirabilis with E. coli, (ii) P. mirabilis with E. faecalis, (iii) P. mirabilis with $M$. morganii, and (iv) M. morganii with $E$. faecalis. A triple-species infection was also conducted for $P$. mirabilis with $E$. faecalis and $M$. morganii, since cocultures involving $E$. faecalis consistently presented the most severe phenotypes in vitro. For each mouse, a 4-mm segment of sterile silicone catheter tubing was inserted into the bladder at the time of inoculation to recapitulate CAUTI, as previously reported (47). Weight loss was monitored daily (see Fig. S2 in the supplemental material), and mice were euthanized at $96 \mathrm{~h}$ postinoculation (hpi) to quantify bacterial burden (Fig. 5 and Fig. S3) and the incidence of bacteremia and macroscopic kidney stones (Table 1). For each infection group, a subset of mice had the entire bladder and both kidneys sectioned and paraffin embedded for assessment of histopathology rather than quantification of bacterial burden (Fig. 5F to $\mathrm{M}$ and Fig. S4).

All mice exhibited weight loss during the first $24 \mathrm{hpi}$, regardless of infection group, and the majority of mice failed to recover weight during the study. The only group of mice that 
A

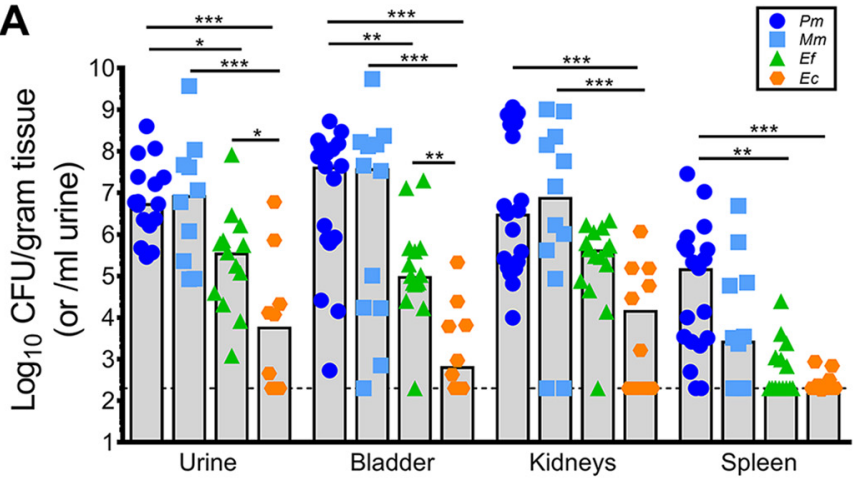

B
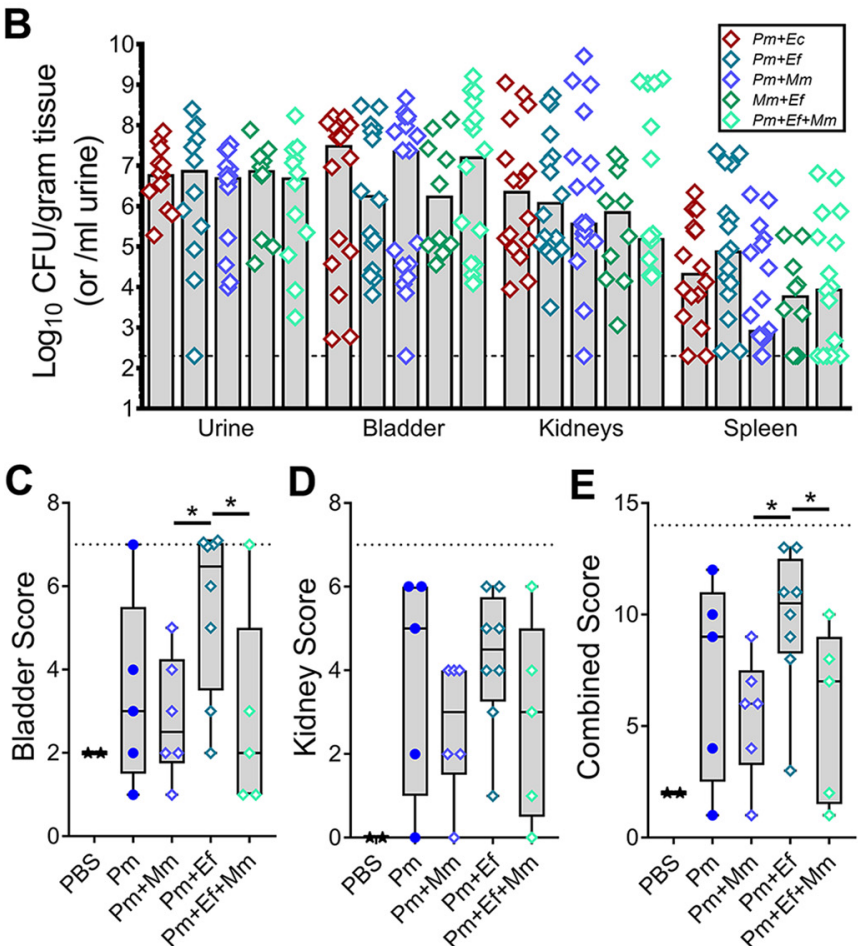

E

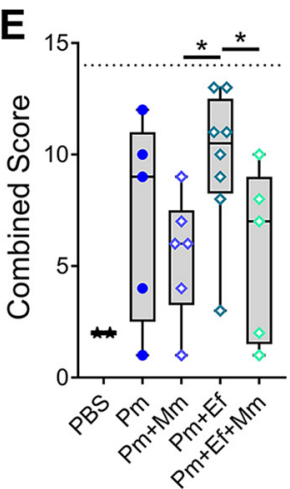

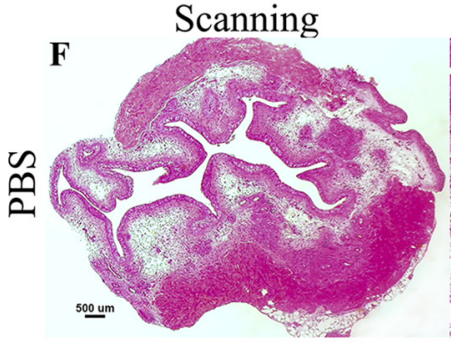
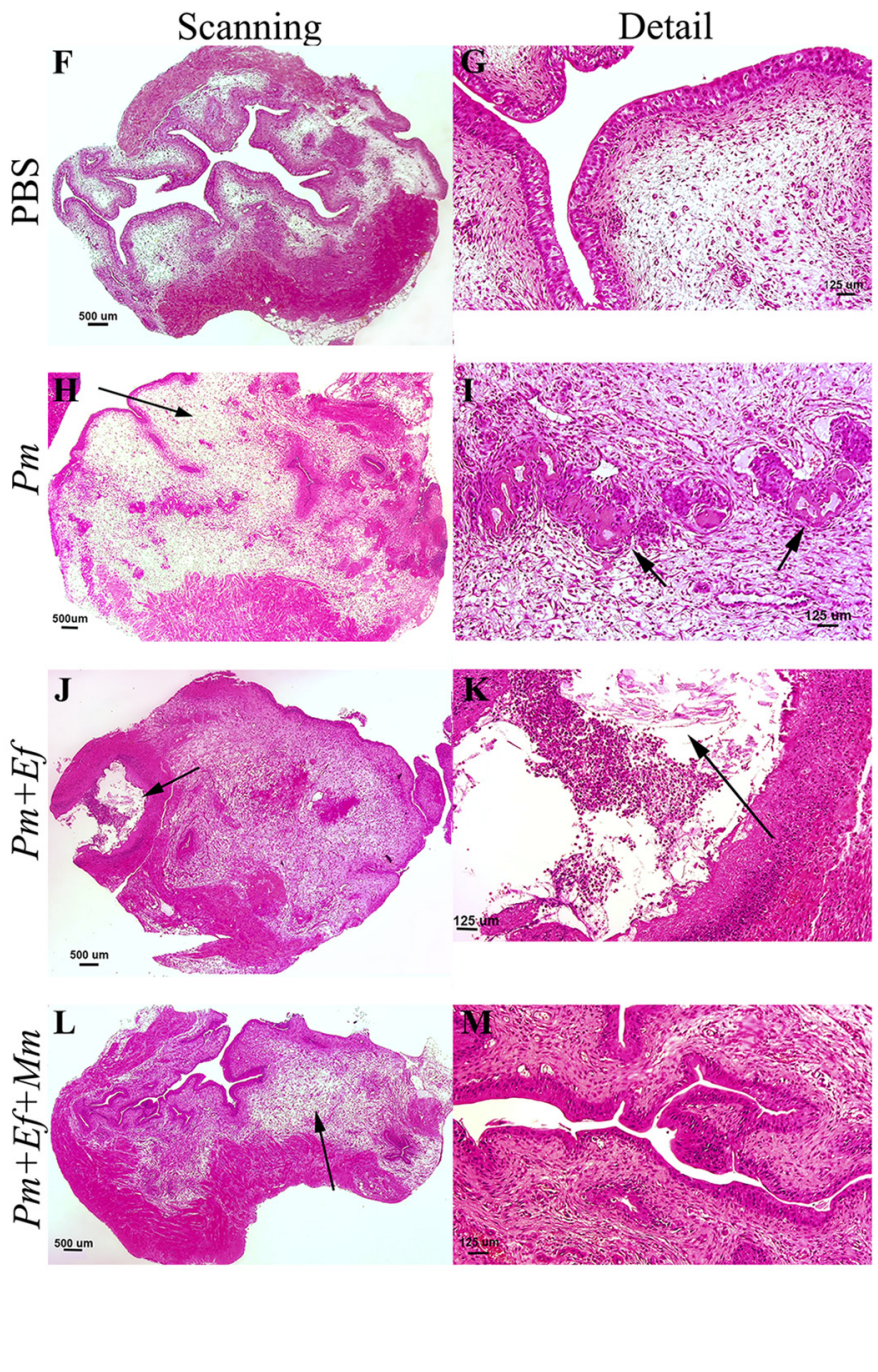

FIG 5 Urinary tract bacterial burden and tissue histopathology during monomicrobial and polymicrobial CAUTI. The log ${ }_{10}$ CFU recovered from the urine, bladder, kidneys, and spleen per gram of tissue or milliliter of urine are shown for monomicrobial infections (A), as are the total CFU recovered from polymicrobial infections (B). Each symbol represents the CFU recovered from a single mouse, gray bars indicate the median, and dashed lines indicate the limit of detection (200 CFU). The number of mice in each infection group is provided in Table 1. For statistical analysis, all CFU data were log ${ }_{10}$ transformed, and significance was assessed by two-way ANOVA with Tukey's multiple-comparison test ( ${ }^{*}, P<0.05 ;{ }^{* *}, P<0.01$; $\left.{ }^{* * *}, P<0.001\right)$. (C to M) Bladder and kidney sections from mice inoculated with PBS $(n=2), P$. mirabilis $(n=5), P$. mirabilis-M. morganii $(n=6), P$. mirabilis-E. faecalis $(n=8)$, or $P$. mirabilis-E. faecalis-M. morganii $(n=5)$ were paraffin embedded, sectioned, and stained with hematoxylin-eosin to assess tissue damage and inflammation using the scoring rubric provided in Table S1. Bladder scores (C), kidney scores (D), and combined scores for bladder and kidney (E) from each individual mouse are shown. ${ }^{*}, P<0.05$ (by nonparametric Mann-Whitney test). ( $F$ to $M$ ) Representative bladder images from select infection groups, including both a low-magnification ( $2 \times$ objective) scanning view (F, H, J, and $\mathrm{L}$ ) and a higher-magnification detail view (G, I, K, and $\mathrm{M})$. (F and $\mathrm{G})$ PBS/mock-infected mice, normal healthy bladder. (H) P. mirabilis monomicrobial infection with severe edema (arrow) and multifocal vasculitis. (I) Detail of vasculitis (arrows) and widespread neutrophilic inflammation. (J) $P$. mirabilis-E. faecalis coinfection showing severe edema, neutrophilic inflammation, and ulceration with embedded calculus (arrow). (K) Detail of calculus (arrow) and surrounding inflammation and necrosis. (L) P. mirabilis-E. faecalis-M. morganii coinfection showing intact mucosa and moderate edema (arrow). (M) Detail of intact mucosa.

exhibited recovery from the initial weight loss were those that received an $E$. coli monomicrobial inoculum (Fig. S2A, C, and H). This is likely because monomicrobial inoculation with $1 \times 10^{5} \mathrm{CFU}$ of $E$. coli resulted in the lowest bacterial burden of all of the infection groups (Fig. 5A), as well as the lowest overall infection severity (Table 1), followed by the $E$. faecalis monomicrobial infection. No other significant differences in weight loss were observed.

Despite differences in the colonization density achieved by each species during monomicrobial infection (Fig. 5A), there were no significant differences in total bacterial 
TABLE 1 CAUTI disease severity ${ }^{a}$

\begin{tabular}{|c|c|c|c|c|c|}
\hline & Number of mice & Bacteremia $^{\mathbf{A}}$ & $P$ value & Urolithiasis $^{B}$ & $P$ value \\
\hline \multicolumn{6}{|c|}{ Monomicrobial Infections } \\
\hline$E c$ & 10 & $20 \%$ & $.001^{*}$ & $0 \%$ & 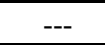 \\
\hline$E f$ & 15 & $47 \%$ & $.040^{*}$ & $0 \%$ & --- \\
\hline$M m$ & 15 & $62 \%$ & .222 & $19 \%$ & .666 \\
\hline$P m$ & 27 & $78 \%$ & --- & $26 \%$ & --- \\
\hline \multicolumn{6}{|c|}{ Polymicrobial Infections } \\
\hline$P m+E c$ & 17 & $82 \%$ & .714 & $29 \%$ & .800 \\
\hline$P m+E f$ & 25 & $96 \%$ & .054 & $56 \%$ & $.027 *$ \\
\hline$P m+E f+M m$ & 21 & $71 \%$ & .614 & $24 \%$ & .867 \\
\hline$P m+M m$ & 26 & $73 \%$ & .691 & $15 \%$ & .344 \\
\hline$E f+M m$ & 10 & $70 \%$ & .624 & $10 \%$ & .296 \\
\hline \multicolumn{6}{|c|}{ Urease-Independent Infections } \\
\hline Pm $\Delta$ ure & 10 & $40 \%$ & $.001 *$ & $0 \%$ & --- \\
\hline$\Delta u r e+E c$ & 10 & $40 \%$ & .999 & $0 \%$ & --- \\
\hline$\Delta u r e+E f$ & 12 & $58 \%$ & .392 & $0 \%$ & --- \\
\hline$\Delta u r e+M m$ & 8 & $37 \%$ & .914 & $12 \%$ & --- \\
\hline$\Delta u r e+E f+M m$ & 12 & $33 \%$ & .746 & $8 \%$ & --- \\
\hline
\end{tabular}

aAbbreviations: Ec, E. coli; Ef, E. faecalis; Mm, M. morganii; Pm, P. mirabilis. (Superscript A) Bacteremia was assessed by spleen CFU above the limit of detection. (Superscript B) Urolithiasis was assessed by the presence of macroscopic kidney stones, as shown in Fig. 4. Severity is expressed relative to $P$. mirabilis (white), with more severe being indicated by orange and less severe being indicated by blue. $P$ values were determined by chi-square analysis versus $P$. mirabilis monomicrobial infection for all groups except for the urease-independent infections, which were compared to $P$. mirabilis $\Delta$ ure infections.

burden within the urinary tract during any of the polymicrobial infections (Fig. 5B). The CFU recovered for each individual bacterial species from the polymicrobial infections are shown across infection groups in Fig. S3A to D and on a per-mouse basis in Fig. S3E to I. P. mirabilis and E. faecalis CFU counts were stable across all infection types (Fig. S3A and $\mathrm{B}), M$. morganii CFU were reduced in the presence of $P$. mirabilis but unaffected by E. faecalis (Fig. S3C), and E. coli CFU were augmented by P. mirabilis (Fig. S3D). Despite these shifts in bacterial burden for each species, the total combined CFU achieved within the urinary tract were consistent across all of the polymicrobial infections (Fig. 5B).

The similarities in total bacterial burden across infection groups are particularly noteworthy considering that there were dramatic differences in disease severity between infection groups (Table 1). P. mirabilis exhibited the greatest disease severity of the monomicrobial infections, with $78 \%$ of mice exhibiting bacteremia and $26 \%$ urolithiasis. Coinfection of $P$. mirabilis with $E$. coli did not significantly impact the incidence of bacteremia or urolithiasis compared to $P$. mirabilis monomicrobial infection, despite the ability of this combination to increase urease activity and cytotoxicity in vitro. In contrast, coinfection of $P$. mirabilis with $E$. faecalis resulted in the most severe disease presentation, since $96 \%$ of these mice exhibited bacteremia (an increase of $18 \%$ relative to $P$. mirabilis monomicrobial infection, $P=0.054$ ) and $56 \%$ exhibited urolithiasis (an increase of $30 \%$ relative to $P$. mirabilis monomicrobial infection, $P=0.027$ ). This coinfection combination also resulted in the greatest incidence of maximum histopathology scores for tissue damage and inflammation in the bladder (Fig. 5C) and the highest combined scores for bladder and kidney damage (Fig. 5E). Representative bladder sections are displayed in Fig. $5 \mathrm{~F}$ to $\mathrm{M}$, and representative kidney sections are displayed in Fig. S4.

Remarkably, the addition of M. morganii to the $P$. mirabilis and E. faecalis coinfection dramatically reduced infection severity to a level that was indistinguishable from that seen in mice infected with $P$. mirabilis alone or from mice coinfected with $P$. mirabilis and $M$. morganii (Table 1). A total of $71 \%$ of mice from the triple-species infection 


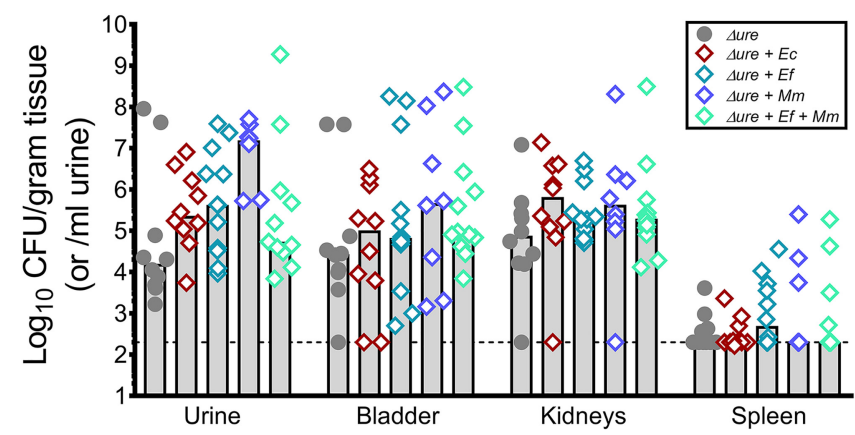

FIG 6 Urinary tract bacterial burden during monomicrobial and polymicrobial infection with $P$. mirabilis $\Delta$ ure. The $\log _{10}$ CFU values recovered from the urine, bladder, kidneys, and spleen per gram of tissue or milliliter of urine are displayed for $P$. mirabilis $\Delta$ ure monomicrobial infection, as are the total CFU recovered from polymicrobial infections. Each symbol represents the CFU recovered from a single mouse, gray bars indicate the median, and dashed lines indicate the limit of detection (200 CFU). The number of mice in each infection group is provided in Table 1. All CFU data were $\log _{10}$ transformed, and no significant differences were identified by two-way ANOVA with Tukey's multiple-comparison test.

exhibited bacteremia $(-25 \%$ compared to $P$. mirabilis- $E$. faecalis coinfection, $P=$ 0.0208 ) and $24 \%$ exhibited urolithiasis ( $-32 \%$ compared to $P$. mirabilis-E. faecalis coinfection, $P=0.0272$ ). The decrease in disease severity was also clearly evident by histopathology, since the triple-species infection resulted in significantly reduced bladder scores and combined scores compared to the $P$. mirabilis-E. faecalis coinfection (Fig. $5 \mathrm{C}$ and $\mathrm{E})$. Thus, the ability of $M$. morganii to dampen enhancement of urease activity and cytotoxicity in vitro does indeed translate to a reduction in infection severity in vivo. Taken together, these data indicate that disease severity during polymicrobial CAUTI is dependent on the effects of the specific coinfecting organisms, rather than bacterial burden alone, and that an organism such as $M$. morganii can reduce infection severity even when present at a low density.

Modulation of polymicrobial infection severity is predominantly mediated through a urease-dependent mechanism. Our prior studies of $P$. mirabilis with $P$. stuartii demonstrated that increased disease severity during coinfection of these species was dependent on the presence of a functional urease operon in $P$. mirabilis and enhancement of urease activity $(47,48)$. We therefore tested the hypothesis that $P$. mirabilis urease activity is required for modulation of disease severity during polymicrobial infection. Mice were inoculated as described above, only using the isogenic urease mutant of $P$. mirabilis HI4320 ( $\Delta$ ure). Fig. S5 shows the percent weight change. Consistent with the prior infections, all mice exhibited weight loss during the first 24 hpi, regardless of infection group. However, as expected, the majority of mice inoculated with the $\Delta$ ure mutant exhibited decreased disease severity compared to infection with the wild-type strain, as indicated by a trend toward weight gain from 72 to $96 \mathrm{hpi}$.

Figure 6 shows the total CFU recovered from mice inoculated with P. mirabilis $\Delta$ ure alone for comparison to each of the polymicrobial infections. The CFU recovered for each individual bacterial species from the polymicrobial infections are shown across infection groups in Fig. S6A to D and on a per-mouse basis in Fig. S6E to H. Similar to infections with wild-type $P$. mirabilis, there were no significant differences in total bacterial burden within the urinary tract between infection groups (Fig. 6). P. mirabilis $\Delta$ ure CFU were again largely similar across all infection types (Fig. S6A). Interestingly, $E$. faecalis CFU were significantly decreased in the urine and kidneys when $P$. mirabilis $\Delta$ ure was present (Fig. S6B), which was not observed during infection with the wild-type strain (Fig. S3B), suggesting that E. faecalis colonization may be perturbed by $P$. mirabilis in the absence of urease activity. M. morganii colonization was again significantly decreased in the urine, bladder, and kidneys when $P$. mirabilis $\Delta$ ure was present, indicating that the impact of $P$. mirabilis on $M$. morganii colonization is urease independent (Fig. S6C). Similarly, E. coli colonization was significantly increased when $P$. 
mirabilis $\Delta$ ure was present, indicating that urease activity is not required for enhancement of $E$. coli colonization during coinfection (Fig. S6D).

Despite these differences in the colonization density achieved by each individual bacterial species during coinfection, the total bacterial burden within the urinary tract again remained consistent across infection groups (Fig. 6). Compared to monomicrobial infection with wild-type $P$. mirabilis, $\Delta$ ure mutant infections exhibited a significant reduction in the incidence of bacteremia (40\% compared to $78 \%, P<0.001$ ), which is consistent with the role of urease activity and urolithiasis in tissue damage and progression to bacteremia $(47,48)$. Even more striking was the observation that disease severity remained consistent between monomicrobial infection with $P$. mirabilis $\Delta u r e$ and each of the polymicrobial infections, in stark contrast to the results obtained with wild-type $P$. mirabilis (Table 1). Thus, while urease-independent interactions still have the potential to influence disease severity during polymicrobial P. mirabilis CAUTI, modulation of urease activity is the main contributor to infection severity in this model system. The mechanism(s) by which $M$. morganii dampens $P$. mirabilis urease activity and prevents enhancement by other bacterial species may therefore elucidate potent inhibitors of infection severity and thus could have clinical utility for CAUTIs involving P. mirabilis.

\section{DISCUSSION}

It is now widely recognized that microbes generally exist in complex polymicrobial communities rather than living in isolation. There are numerous experimental and clinical examples of polymicrobial interactions increasing disease severity compared to monomicrobial infection (17-20), as well as examples of interactions that attenuate disease severity (21). However, the clinical significance of polymicrobial interactions is less clear in the context of bacteriuria, particularly for individuals with indwelling urinary catheters. In the present study, we show that bacterial species colonizing the catheterized urinary tract can both enhance (E. faecalis, E. coli, and P. stuartii) and dampen (M. morganii) disease severity by exerting an effect on $P$. mirabilis and that this process does not require physical contact or close proximity of the bacteria. We further demonstrate that the predominant cause of disease severity is modulation of $P$. mirabilis urease activity, but additional urease-independent factors also contribute to cytotoxicity to host cells.

M. morganii is a common constituent of the intestinal tract and considered to be a rare opportunistic pathogen, mainly causing nosocomial urinary tract infections in elderly catheterized individuals (53). This species has occasionally been identified as a cause of severe UTI-related complications such as sepsis and bacteremia, although it is less common than P. mirabilis or E. coli and more likely to be present during polymicrobial sepsis than as the sole causative agent of disease (54). Thus, severe disease manifestations, such as entry of $M$. morganii to the bloodstream, are likely facilitated by other bacterial species, particularly within the urinary tract, where M. morganii is generally present during polymicrobial infection $(6,55)$. Patients experiencing $M$. morganii sepsis are also more likely than patients with $E$. coli to have multiple comorbidities and reduced functional status (54), underscoring the opportunistic nature of this organism. It is therefore fascinating that this rare opportunist has such a dramatic impact on the pathogenesis of polymicrobial CAUTI involving $P$. mirabilis. Our results clearly demonstrate that $M$. morganii dampens $P$. mirabilis urease activity and even prevents enhancement by other bacterial species, which is consistent with prior reports of $M$. morganii reducing catheter blockage and encrustation by $P$. mirabilis in vitro (24). However, our study further extends these observations to show that $M$. morganii significantly attenuates polymicrobial CAUTI.

Our experimental findings have important clinical implications, particularly for patient populations requiring long-term catheterization. Approximately $50 \%$ of individuals catheterized for 28 days or longer experience catheter blockage and/or encrustation from crystalline deposits, the vast majority of which are the result of $P$. mirabilis urease activity $(10,12,56,57)$. These crystalline deposits accumulate on the catheter 


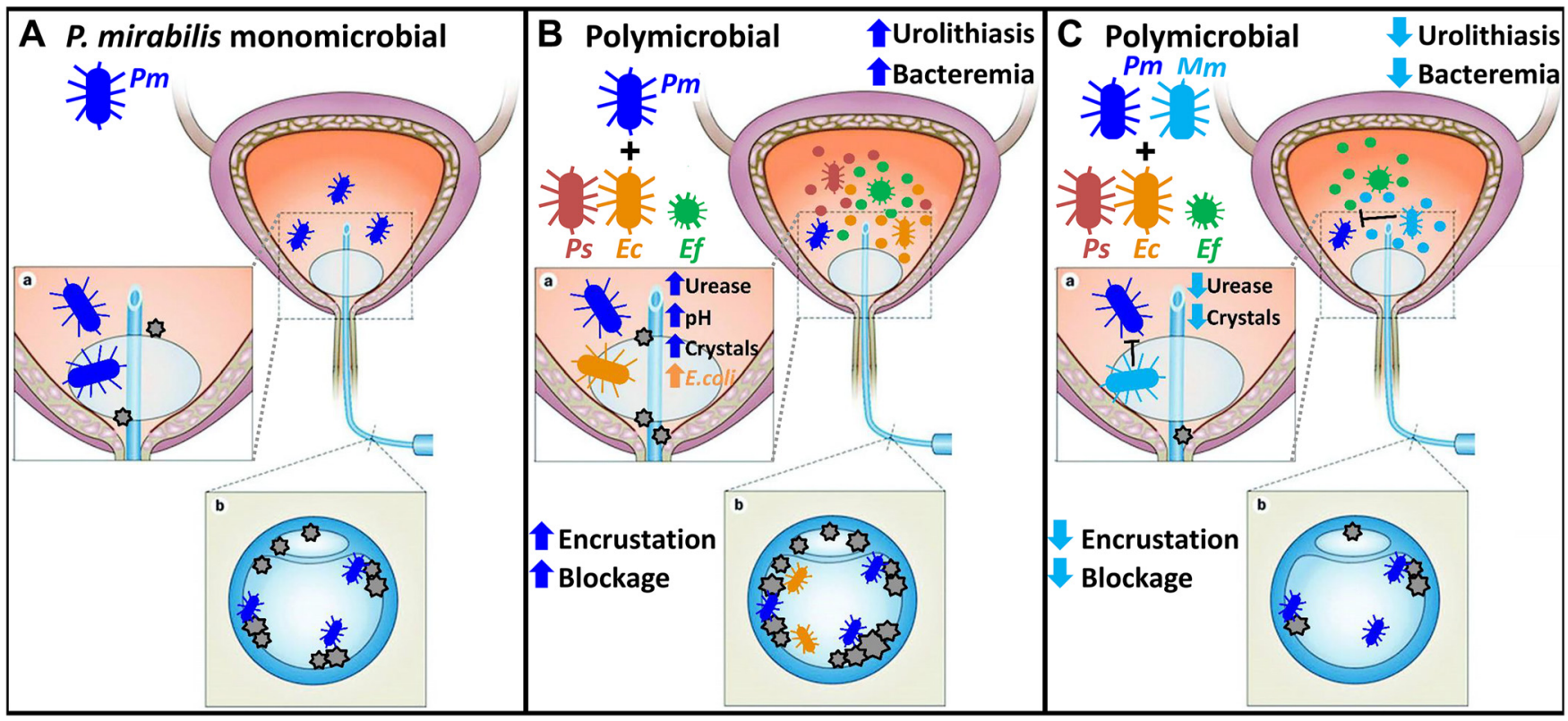

FIG 7 Implications of polymicrobial interactions during CAUTI. (A) P. mirabilis (blue) monomicrobial colonization. Urease activity from this bacterial species results in crystalline deposits in the bladder (a, bladder inset) and on the catheter (b, catheter inset), promoting bacterial colonization and in some cases bacteremia. (B) Polymicrobial colonization involving P. mirabilis and P. stuartii (maroon), E. coli (orange), or E. faecalis (green). Cocolonizing species secrete factors that act on $P$. mirabilis to enhance urease activity, thereby raising urine $\mathrm{pH}$ and increasing crystal formation, which increases the risk of catheter encrustation and blockage. Cocolonization with E. faecalis also increases the risk of urolithiasis and bacteremia, and E. coli colonization is augmented by the presence of $P$. mirabilis. (C) Polymicrobial colonization involving P. mirabilis and M. morganii (light blue) with P. stuartii, E. coli, or E. faecalis. M. morganii secretes factors that act on $P$. mirabilis to dampen urease activity and prevent enhancement by other species, such as $E$. faecalis, thereby reducing the formation of crystals and the risk of catheter encrustation and blockage. The presence of $M$. morganii also decreases risk of urolithiasis and bacteremia during coinfection with $P$. mirabilis and $E$. faecalis. (Catheterized bladder image adapted from reference 78 and from reference 79 with permission of the publisher.)

surface and facilitate biofilm formation (58), a bacterial mode of growth that is associated with immune evasion and antimicrobial resistance (59). Many of the ureasepositive organisms that colonize urinary catheters can persist within a catheterized host for months, even after antibiotic treatment and catheter changes (14). Thus, interactions between bacteria within the catheterized bladder and in catheter biofilms have the potential to significantly impact catheter encrustation, tissue damage, urolithiasis, and dissemination to the kidneys and bloodstream.

The implications of our findings, in this context, are depicted in Fig. 7. When $P$. mirabilis is present in the bladder in the absence of other bacterial species, it is capable of causing stone formation and crystalline catheter biofilms, ascending to the kidneys, and potentially disseminating to the bloodstream (Fig. 7A). When an enhancing species is present, such as $P$. stuartii, E. coli, or E. faecalis, secreted factors produced by these organisms act on $P$. mirabilis to increase urease activity and cytotoxicity. This leads to an increase in tissue damage, urine $\mathrm{pH}$ and crystalline deposits, and catheter encrustation and blockage, in addition to promoting urolithiasis and bacteremia. Bladder cocolonization of $P$. mirabilis with $E$. coli also bolsters $E$. coli CFU, which may potentiate its impact on P. mirabilis (Fig. 7B). However, when M. morganii is present, it secretes factors that counteract the effects of these other microbes on $P$. mirabilis urease activity and cytotoxicity, thereby reducing crystal formation, catheter encrustation and blockage, urolithiasis, and bacteremia (Fig. 7C). Considering the level of activity present in cell-free urine supernatants of these organisms, it is likely that if any of these modulatory organisms are present in the bladder, they would exert an impact on catheterresident $P$. mirabilis.

A few other dual-species polymicrobial combinations have been characterized in murine models of UTI or CAUTI, although most studies have focused on the impact of coinfection on colonization rather than disease severity. For instance, E. faecalis and Streptococcus agalactiae are both capable of promoting $E$. coli colonization by sup- 
pressing the host immune response $(60,61)$. Coinfection of $E$. coli with $P$. mirabilis promotes colonization of both species during uncomplicated UTI through a dampening of the immune response (62), although only E. coli received a benefit from cocolonization in our CAUTI model. Coinfection of $P$. mirabilis with $P$. stuartii has no impact on the bacterial burden of either species within the urinary tract, but their interactions actually promote a potent proinflammatory response, greater tissue damage, and an increased incidence of severe disease during both UTI and CAUTI $(47,48)$. This is likely also the case for coinfection of $P$. mirabilis with $E$. faecalis, given the increase in cytotoxicity that occurs in vitro and the incidence of bacteremia in our CAUTI model. An increased likelihood of severe disease has also been observed during coinfection of $P$. mirabilis with Staphylococcus saprophyticus (63), as well as for P. aeruginosa with $E$. faecalis (64).

In contrast to these infection-promoting interactions, there are limited examples of polymicrobial interactions that attenuate disease severity during UTI or CAUTI. Production of an iron-binding factor by commensal $E$. coli and other Enterobacteriaceae antagonizes iron acquisition by $P$. aeruginosa, thereby limiting growth and possibly survival in the catheterized bladder (65). Other commensal E. coli strains have also been shown to inhibit growth and catheter colonization by traditional uropathogens in vitro $(66,67)$. Thus, our observation of disease modulation by $M$. morganii is consistent with the idea that commensal or nontraditional pathogens can hinder the establishment of infection and disease severity by other pathogens.

In conclusion, our results indicate that further assessments of the clinical significance of polymicrobial bacteriuria and CAUTI need to consider the exact combination of bacterial species that are present, in addition to Gram-positive versus Gram-negative and bacterial burden within the sample. Such interactions may be difficult to capture from single urine samples if colonization of the disease-modulating organism is impacted during coinfection, as is the case for M. morganii and E. coli. However, a deeper understanding of the importance of these interactions is necessary to help guide novel treatment strategies. Furthermore, identification of $M$. morganii secreted products that modulate $P$. mirabilis pathogenicity has the potential to lead to new therapeutic treatments for CAUTI. The abundance of urease-dampening and urease-enhancing factors during asymptomatic colonization may also have predictive value for patient outcomes in long-term catheterization.

\section{MATERIALS AND METHODS}

Study approval. Animal protocols were approved by the Institutional Animal Care and Use Committee of the University at Buffalo (MIC31107Y), in accordance with the Office of Laboratory Animal Welfare, the U.S. Department of Agriculture, and the Association for Assessment and Accreditation of Laboratory Animal Care. Mice were anesthetized with a weight-appropriate dose $(0.1 \mathrm{ml}$ for a mouse weighing $20 \mathrm{~g}$ ) of ketamine/xylazine ( 80 to $120 \mathrm{mg} / \mathrm{kg}$ ketamine and 5 to $10 \mathrm{mg} / \mathrm{kg}$ xylazine) by intraperitoneal injection and euthanized by inhalant anesthetic overdose with vital organ removal.

Bacterial strains and growth conditions. Proteus mirabilis HI4320, Providencia stuartii BE2467, and Morganella morganii TA43 were isolated from the urine of catheterized patients in a chronic care facility in Maryland (12). Escherichia coli CFT073 was isolated from a patient hospitalized for acute pyelonephritis (68). Enterococcus faecalis 3143 was isolated from the urine of a catheterized nursing home resident in Michigan (47). P. mirabilis $\Delta$ ure refers to $P$. mirabilis HI4320 lacking urease activity due to an insertional disruption of the ureF gene, and $P$. stuartii $\Delta$ ure refers to $P$. stuartii BE2467 lacking the portion of its plasmid that carries the urease operon $(47,69,70)$. Bacteria were routinely cultured at $37^{\circ} \mathrm{C}$ with aeration in $5 \mathrm{ml}$ of lysogeny broth (LB) (10 g/liter tryptone, $5 \mathrm{~g} /$ liter yeast extract, $0.5 \mathrm{~g} /$ liter NaCl) or on LB medium solidified with $1.5 \%$ agar. Enterococcus faecalis isolates were cultured in brain heart infusion broth (BHI). LB medium was supplemented with $25 \mu \mathrm{g} / \mathrm{ml}$ kanamycin, $20 \mu \mathrm{g} / \mathrm{ml}$ chloramphenicol, $25 \mu \mathrm{g} / \mathrm{ml}$ ampicillin, $2.5 \mu \mathrm{g} / \mathrm{ml}$ tetracycline, or $100 \mu \mathrm{g} / \mathrm{ml}$ streptomycin to distinguish between species.

Urine growth curves. Pooled, filter-sterilized human urine from female donors (Cone Bioproducts) was diluted 1:1 with sterile saline to achieve a uniform specific gravity of $\sim 1.005$. Overnight cultures of bacterial species were diluted 1:100 into $5 \mathrm{ml}$ of urine for monomicrobial growth curves. For cocultures each bacterial species was diluted 1:200 to maintain the same total starting CFU, and for triple cultures each bacterial species was diluted 1:300. Bacterial burden was assessed by plating for CFU. To distinguish between bacterial species, samples were plated as follows: (i) $P$. mirabilis-E. faecalis coinfection samples were plated on plain LB medium (total CFU) and BHI with streptomycin (E. faecalis CFU), (ii) P. mirabilis-M. morganii coinfection samples were plated on plain LB medium (total CFU) and LB medium with ampicillin (M. morganii CFU), (iii) P. mirabilis-E. coli coinfection samples were plated on plain LB medium (total CFU) 
and LB medium with tetracycline (P. mirabilis CFU), (iv) $E$. faecalis-M. morganii coinfection samples were plated on plain LB medium (total CFU) and BHI with streptomycin (E. faecalis), and (v) P. mirabilis-E. faecalis-M. morganii triple-infection samples were plated on plain LB (total CFU), LB medium with ampicillin (M. morganii CFU), and BHI with streptomycin (E. faecalis CFU).

Urease assay. A previously described alkalimetric screen was used to measure urease activity in whole bacterial cells (47). Briefly, bacterial strains were cultured to mid-log phase (OD at $600 \mathrm{~nm}$ $\left[\mathrm{OD}_{600}\right]=0.5$ ), centrifuged to pellet, and resuspended in filter-sterilized human urine (using a batch pool from deidentified donors, purchased from Cone Bioproducts). Resuspended cultures were incubated individually at $37^{\circ} \mathrm{C}$ with aeration or mixed in equal proportions to generate cocultures. After $90 \mathrm{~min}$, the cultures were centrifuged to pellet, and supernatants were passaged through a $0.22-\mu \mathrm{m}$-pore-size filter to generate cell-free spent urine supernatants for determination of $P$. mirabilis urease activity. For measurement of urease enhancement in spent urine supernatants, $P$. mirabilis $\mathrm{HI} 4320$ was cultured for $\sim 16 \mathrm{~h}$ at $37^{\circ} \mathrm{C}$ with aeration in $5 \mathrm{ml}$ of $\mathrm{LB}$, centrifuged to pellet, and resuspended in $0.9 \%$ sterile saline. The urine supernatants were dispensed into replicated wells of a 96-well plate, supplemented with a phenol red and urea mixture to a final concentration of $0.001 \%$ (wt/vol) phenol red and $500 \mathrm{mM}$ urea, and inoculated with $20 \mu \mathrm{l}$ of the bacterial saline suspension. The $\mathrm{OD}_{562}$ was measured every $60 \mathrm{~s}$ for $2 \mathrm{~h}$ using a Synergy $\mathrm{H} 1$ (BioTek). The urease activity was expressed as the mean change in $\mathrm{OD}$ per minute $(\mathrm{mOD} / \mathrm{min})$ for the linear portion of the activity curve, as calculated by the Gen5 software (BioTek).

Cytotoxicity. Toxicity of bacterial species independently or during coculture was estimated by an LDH release cytotoxicity assay, as previously described $(47,71)$. HEK293 cells were purchased from the American Type Culture Collection in 2018 and used for cytotoxicity studies after $\leq 3$ passages. Briefly, bacteria were cultured in LB medium to mid-log phase $\left(\mathrm{OD}_{600}=0.5\right)$, centrifuged to pellet, and resuspended in filter-sterilized human urine to induce urease activity. Resuspended cultures were incubated for $60 \mathrm{~min}$ at $37^{\circ} \mathrm{C}$. Cultures were then pelleted, washed twice, resuspended in Dulbecco modified Eagle medium without supplements or antibiotics to a concentration of $2 \times 10^{7} \mathrm{CFU} / \mathrm{ml}$, and added to a monolayer of low-passage-number HEK293 cells at a multiplicity of infection of approximately 100:1. After a 4-h incubation at $37^{\circ} \mathrm{C}$ and $5 \% \mathrm{CO}_{2}$, the amount of $\mathrm{LDH}$ released into the supernatant was measured using an LDH cytotoxicity assay kit (Cayman Chemical). Treatment with $9 \%$ Triton X-100 for the 4-h incubation was used as a positive control for maximum lysis, and treatment with sterile urine alone was used as a negative control. The level of HEK293 lysis under each treatment condition is expressed relative to the level for $P$. mirabilis $\Delta$ ure. For all dual-species and triple-species cultures, a predicted (P) level of cytotoxicity was calculated based on the proportion of each bacterial species in the culture (determined by differential plating for $\mathrm{CFU}$ ) and the relative cytotoxicity of monomicrobial cultures for each species.

Mouse model of CAUTI. Infection studies were carried out as previously described (47). Briefly, bacteria were cultured overnight in LB medium, washed in phosphate-buffered saline (PBS; $0.128 \mathrm{M}$ $\mathrm{NaCl}, 0.0027 \mathrm{M} \mathrm{KCl}[\mathrm{pH} 7.4])$, adjusted to $2 \times 10^{8} \mathrm{CFU} / \mathrm{ml}\left(\mathrm{OD}_{600}=0.2\right.$ for $P$. mirabilis, $P$. stuartii, and M. morganii, and $\mathrm{OD}_{600}=0.4$ for E. coli and E. faecalis), and diluted 1:100 to achieve an inoculum of $2 \times 10^{6} \mathrm{CFU} / \mathrm{ml}$. Female CBA/J mice at 6 to 8 weeks of age (Jackson) were inoculated transurethrally with $50 \mu \mathrm{l}$ of $2 \times 10^{6} \mathrm{CFU} / \mathrm{ml}\left(1 \times 10^{5} \mathrm{CFU} /\right.$ mouse) of a single bacterial species (monomicrobial), a 1:1 mixture of two species for coinfections, or a 1:1:1 mixture of $P$. mirabilis with two other species for polymicrobial infections. In all cases, a 4-mm segment of sterile silicone tubing $(0.64 \mathrm{~mm}$ [outer diameter], $0.30 \mathrm{~mm}$ [inner diameter]; Braintree Scientific, Inc.) was carefully advanced into the bladder during inoculation as described elsewhere $(47,72-74)$. Mice were euthanized $96 \mathrm{hpi}$, and bladders, kidneys, and spleens were harvested into 5-ml Eppendorf tubes containing $1 \mathrm{ml}$ of PBS. Gross inspection of kidneys was performed to assess macroscopic urolithiasis. Tissues were homogenized using a Bullet Blender 5 Gold (Next Advance) and plated using an EddyJet 2 spiral plater (Neutec Group) for determination of CFU using a ProtoCOL 3 automated colony counter (Synbiosis). To distinguish between bacterial species, samples were plated as follows: (i) P. mirabilis-E. faecalis coinfection samples were plated on plain LB medium (total $\mathrm{CFU}$ ) and BHI with streptomycin ( $E$. faecalis CFU), (ii) $P$. mirabilis-M. morganii coinfection samples were plated on plain LB medium (total CFU) and LB medium with ampicillin (M. morganii CFU), (iii) $P$. mirabilis-E. coli coinfection samples were plated on plain LB medium (total CFU) and LB medium with tetracycline ( $P$. mirabilis CFU), (iv) E. faecalis-M. morganii coinfection samples were plated on plain LB medium (total CFU) and BHI with streptomycin (E. faecalis), and (v) P. mirabilis-E. faecalis-M. morganii triple infection samples were plated on plain LB medium (total CFU), LB medium with ampicillin (M. morganii CFU), and BHI with streptomycin (E. faecalis CFU).

Pathological evaluation. A subset of mice from each infection group were selected for histopathological examination rather than determination of bacterial burden. For these mice, bladders were cut longitudinally and each kidney was transversely. Organs were preserved in $10 \%$ formalin, embedded in paraffin, sectioned, and stained with hematoxylin and eosin. Sections were examined microscopically and scored in a blinded fashion by a veterinary pathologist to determine the severity and extent of inflammation and lesions using an adaptation of a previously developed semiquantitative scoring system $(47,71,75-77)$. The scoring rubric is provided in Table S1 in the supplemental material.

Statistics. Normalcy was assessed for all data sets by the Shapiro-Wilk and D'Agostino-Pearson normality tests. Significance was assessed using two-way analysis of variance (ANOVA), a nonparametric Mann-Whitney test, an unpaired $t$ test, or a chi-square test, as indicated in the figure legends. All $P$ values are two tailed at a 95\% confidence interval. All analyses were performed using Prism, version 7.03 (GraphPad Software, San Diego, CA). 


\section{SUPPLEMENTAL MATERIAL}

Supplemental material is available online only.

SUPPLEMENTAL FILE 1, PDF file, 7.5 MB.

\section{ACKNOWLEDGMENTS}

We thank members of the Department of Microbiology and Immunology in the Jacobs School of Medicine and Biomedical Sciences at the University at Buffalo for helpful comments and critiques.

This study was supported by the National Institutes of Health (grant R00 DK105205 to C.E.A.). The sponsors were not involved in the study design, methods, subject recruitment, data collections, analysis, or preparation of the paper. The content is solely the responsibility of the authors and does not necessarily represent the official views of the funders.

\section{REFERENCES}

1. Hooton TM, Bradley SF, Cardenas DD, Colgan R, Geerlings SE, Rice JC, Saint S, Schaeffer AJ, Tambayh PA, Tenke P, Nicolle LE. 2010. Diagnosis, prevention, and treatment of catheter-associated urinary tract infection in adults: 2009 International Clinical Practice Guidelines from the Infectious Diseases Society of America. Clin Infect Dis 50:625-663. https:// doi.org/10.1086/650482.

2. Nicolle LE. 2012. Urinary catheter-associated infections. Infect Dis Clin North Am 26:13-27. https://doi.org/10.1016/j.idc.2011.09.009.

3. Dudeck MA, Edwards JR, Allen-Bridson K, Gross C, Malpiedi PJ, Peterson KD, Pollock DA, Weiner LM, Sievert DM. 2015. National Healthcare Safety Network report, data summary for 2013, device-associated module. Am J Infect Control 43:206-221. https://doi.org/10.1016/j.ajic.2014.11.014.

4. Hollenbeak CS, Schilling AL. 2018. The attributable cost of catheterassociated urinary tract infections in the United States: a systematic review. Am J Infect Control 46:751-757. https://doi.org/10.1016/j.ajic 2018.01.015.

5. Nicolle LE. 2005. Catheter-related urinary tract infection. Drugs Aging 22:627-639. https://doi.org/10.2165/00002512-200522080-00001.

6. Armbruster CE, Prenovost K, Mobley HLT, Mody L. 2017. How often do clinically diagnosed catheter-associated urinary tract infections in nursing home residents meet standardized criteria? J Am Geriatr Soc 65: 395-401. https://doi.org/10.1111/jgs.14533.

7. Dedeic-Ljubovic A, Hukic M. 2009. Catheter-related urinary tract infection in patients suffering from spinal cord injuries. Bosn J Basic Med Sci 9:2-9. https://doi.org/10.17305/bjbms.2009.2849.

8. Ronald A. 2003. The etiology of urinary tract infection: traditional and emerging pathogens. Dis Mon 49:71-82. https://doi.org/10.1067/mda .2003.8.

9. Siegman-Igra Y, Kulka T, Schwartz D, Konforti N. 1994. Polymicrobial and monomicrobial bacteraemic urinary tract infection. J Hosp Infect 28: 49-56. https://doi.org/10.1016/0195-6701(94)90152-x.

10. Kunin CM. 1989. Blockage of urinary catheters: role of microorganisms and constituents of the urine on formation of encrustations. J Clin Epidemiol 42:835-842. https://doi.org/10.1016/0895-4356(89)90096-6.

11. Rahav G, Pinco E, Silbaq F, Bercovier H. 1994. Molecular epidemiology of catheter-associated bacteriuria in nursing home patients. J Clin Microbiol 32:1031-1034.

12. Mobley HLT, Warren JW. 1987. Urease-positive bacteriuria and obstruction of long-term urinary catheters. J Clin Microbiol 25:2216-2217.

13. Breitenbucher RB. 1984. Bacterial changes in the urine samples of patients with long-term indwelling catheters. Arch Intern Med 144: 1585-1588. https://doi.org/10.1001/archinte.1984.00350200077013.

14. Warren JW, Tenney JH, Hoopes JM, Muncie HL, Anthony WC. 1982. A prospective microbiologic study of bacteriuria in patients with chronic indwelling urethral catheters. J Infect Dis 146:719-723. https://doi.org/ 10.1093/infdis/146.6.719.

15. Kline KA, Lewis AL. 2016. Gram-positive uropathogens, polymicrobial urinary tract infection, and the emerging microbiota of the urinary tract. Microbiol Spectr 4:UTI-0012-2012. https://doi.org/10.1128/microbiolspec .UTI-0012-2012.

16. Hooton TM. 2012. Clinical practice: uncomplicated urinary tract infection. $\mathrm{N}$ Engl J Med 366:1028-1037. https://doi.org/10.1056/NEJMcp1104429.

17. Murray JL, Connell JL, Stacy A, Turner KH, Whiteley M. 2014. Mechanisms of synergy in polymicrobial infections. J Microbiol 52:188-199. https:// doi.org/10.1007/s12275-014-4067-3.

18. Nair N, Biswas R, Götz F, Biswas L. 2014. Impact of Staphylococcus aureus on pathogenesis in polymicrobial infections. Infect Immun 82: 2162-2169. https://doi.org/10.1128/IAl.00059-14.

19. Short FL, Murdoch SL, Ryan RP. 2014. Polybacterial human disease: the ills of social networking. Trends Microbiol 22:508-516. https://doi.org/ 10.1016/j.tim.2014.05.007.

20. Tay WH, Chong KK, Kline KA. 2016. Polymicrobial-host interactions during infection. J Mol Biol 428:3355-3371. https://doi.org/10.1016/j.jmb .2016.05.006.

21. Hajishengallis G, Lamont RJ. 2016. Dancing with the stars: how choreographed bacterial interactions dictate nososymbiocity and give rise to keystone pathogens, accessory pathogens, and pathobionts. Trends Microbiol 24:477-489. https://doi.org/10.1016/j.tim.2016.02.010.

22. Armbruster CE, Prenovost K, Mobley HLT, Mody L. 2016. How often do clinically diagnosed catheter-associated urinary tract infections in nursing home residents meet standardized criteria? J Am Geriatr Soc 65: 395-401. https://doi.org/10.1111/jgs.14533.

23. Fourcade C, Canini L, Lavigne J-P, Sotto A. 2015. A comparison of monomicrobial versus polymicrobial Enterococcus faecalis bacteriuria in a French university hospital. Eur J Clin Microbiol Infect Dis 34:1667-1673. https://doi.org/10.1007/s10096-015-2403-0.

24. Macleod SM, Stickler DJ. 2007. Species interactions in mixed-community crystalline biofilms on urinary catheters. J Med Microbiol 56:1549-1557. https://doi.org/10.1099/jmm.0.47395-0.

25. Armbruster CE, Mobley HL. 2015. Proteus species. In Yu VL (ed), Antimicrobial therapy and vaccines. E-Sun Technologies, Pittsburgh, PA. http:// www.antimicrobe.org/b226.asp.

26. Korytny A, Riesenberg K, Saidel-Odes L, Schlaeffer F, Borer A. 2016. Bloodstream infections caused by multidrug-resistant Proteus mirabilis: epidemiology, risk factors, and impact of multidrug resistance. Infect Dis (Lond) 48:428-431. https://doi.org/10.3109/23744235.2015.1129551.

27. Kanayama A, Kobayashi I, Shibuya K. 2015. Distribution and antimicrobial susceptibility profile of extended-spectrum $\beta$-lactamase-producing Proteus mirabilis strains recently isolated in Japan. Int J Antimicrob Agents 45:113-118. https://doi.org/10.1016/j.ijantimicag.2014.06.005.

28. Wang J-T, Chen P-C, Chang S-C, Shiau Y-R, Wang H-Y, Lai J-F, Huang I-W, Tan M-C, Lauderdale T-L. 2014. Antimicrobial susceptibilities of Proteus mirabilis: a longitudinal nationwide study from the Taiwan surveillance of antimicrobial resistance (TSAR) program. BMC Infect Dis 14:486. https://doi.org/10.1186/1471-2334-14-486.

29. Luzzaro F, Ortisi G, Larosa M, Drago M, Brigante G, Gesu G. 2011. Prevalence and epidemiology of microbial pathogens causing bloodstream infections: results of the OASIS multicenter study. Diagn Microbiol Infect Dis 69:363-369. https://doi.org/10.1016/j.diagmicrobio.2010 .10 .016 .

30. Tumbarello M, Trecarichi EM, Fiori B, Losito AR, D'Inzeo T, Campana L, Ruggeri A, Di Meco E, Liberto E, Fadda G, Cauda R, Spanu T. 2012. Multidrug-resistant Proteus mirabilis bloodstream infections: risk factors and outcomes. Antimicrob Agents Chemother 56:3224-3231. https://doi .org/10.1128/AAC.05966-11.

31. Endimiani A, Luzzaro F, Brigante G, Perilli M, Lombardi G, Amicosante G, Rossolini GM, Toniolo A. 2005. Proteus mirabilis bloodstream infections: 
risk factors and treatment outcome related to the expression of extended-spectrum $\beta$-lactamases. Antimicrob Agents Chemother 49: 2598-2605. https://doi.org/10.1128/AAC.49.7.2598-2605.2005.

32. Ahn JY, Ann HW, Jeon Y, Ahn MY, Oh DH, Kim YC, Kim EJ, Song JE, Jung IY, Kim MH, Jeong W, Ku NS, Jeong SJ, Choi JY, Yong D, Song YG, Kim JM. 2017 The impact of production of extended-spectrum $\beta$-lactamases on the 28day mortality rate of patients with Proteus mirabilis bacteremia in Korea. BMC Infect Dis 17:327. https://doi.org/10.1186/s12879-017-2431-8.

33. Wang J, Foxman B, Mody L, Snitkin ES. 2017. Network of microbial and antibiotic interactions drive colonization and infection with multidrugresistant organisms. Proc Natl Acad Sci U S A 114:10467-10472. https:// doi.org/10.1073/pnas.1710235114.

34. de Vos MGJ, Zagorski M, McNally A, Bollenbach T. 2017. Interaction networks, ecological stability, and collective antibiotic tolerance in polymicrobial infections. Proc Natl Acad Sci U S A 114:10666-10671. https:// doi.org/10.1073/pnas.1713372114.

35. Griffith DP, Musher DM, Itin C. 1976. Urease: the primary cause of infection-induced urinary stones. Invest Urol 13:346-350.

36. Nicholson EB, Concaugh EA, Mobley HL. 1991. Proteus mirabilis urease: use of a ureA-lac $Z$ fusion demonstrates that induction is highly specific for urea. Infect Immun 59:3360-3365.

37. Coker C, Poore CA, Li X, Mobley H. 2000. Pathogenesis of Proteus mirabilis urinary tract infection. Microbes Infect 2:1497-1505. https://doi .org/10.1016/S1286-4579(00)01304-6.

38. Li X, Zhao H, Lockatell CV, Drachenberg CB, Johnson DE, Mobley H. 2002. Visualization of Proteus mirabilis within the matrix of urease-induced bladder stones during experimental urinary tract infection. Infect Immun 70:389-394. https://doi.org/10.1128/iai.70.1.389-394.2002.

39. Foxman B, Brown P. 2003. Epidemiology of urinary tract infections: transmission and risk factors, incidence, and costs. Infect Dis Clin North Am 17:227-241. https://doi.org/10.1016/S0891-5520(03)00005-9.

40. Mylotte JM, Tayara A, Goodnough S. 2002. Epidemiology of bloodstream infection in nursing home residents: evaluation in a large cohort from multiple homes. Clin Infect Dis 35:1484-1490. https://doi.org/10.1086/ 344649.

41. Muder RR, Brennen C, Wagener MM, Goetz AM. 1992. Bacteremia in a long-term-care facility: a five-year prospective study of 163 consecutive episodes. Clin Infect Dis 14:647-654. https://doi.org/10.1093/clinids/14 .3.647.

42. Setia U, Serventi I, Lorenz P. 1984. Bacteremia in a long-term care facility: spectrum and mortality. Arch Intern Med 144:1633-1635. https://doi .org/10.1001/archinte.1984.00350200143021.

43. Rudman D, Hontanosas A, Cohen Z, Mattson DE. 1988. Clinical correlates of bacteremia in a Veterans Administration extended care facility. J Am Geriatr Soc 36:726-732. https://doi.org/10.1111/j.1532-5415.1988.tb07175.x.

44. Kim BN, Kim NJ, Kim MN, Kim YS, Woo JH, Ryu J. 2003. Bacteraemia due to tribe Proteeae: a review of 132 cases during a decade (1991-2000). Scand J Infect Dis 35:98-103. https://doi.org/10.1080/0036554021000027015.

45. Lubart E, Segal R, Haimov E, Dan M, Baumoehl Y, Leibovitz A. 2011. Bacteremia in a multilevel geriatric hospital. J Am Med Dir Assoc 12: 204-207. https://doi.org/10.1016/j.jamda.2010.02.017.

46. Nicolle LE. 2001. Urinary tract pathogens in complicated infection and in elderly individuals. J Infect Dis 183(Suppl 1):S5-S8. https://doi.org/10.1086/ 318844.

47. Armbruster CE, Smith SN, Johnson AO, DeOrnellas V, Eaton KA, Yep A, Mody L, Wu W, Mobley H. 2017. The pathogenic potential of Proteus mirabilis is enhanced by other uropathogens during polymicrobial urinary tract infection. Infect Immun 85:e00808-16. https://doi.org/10.1128/IAl.00808-16.

48. Armbruster CE, Smith SN, Yep A, Mobley H. 2014. Increased incidence of urolithiasis and bacteremia during Proteus mirabilis and Providencia stuartii coinfection due to synergistic induction of urease activity. J Infect Dis 209:1524-1532. https://doi.org/10.1093/infdis/jit663.

49. Hu LT, Nicholson EB, Jones BD, Lynch MJ, Mobley HL. 1990. Morganella morganii urease: purification, characterization, and isolation of gene sequences. J Bacteriol 172:3073-3080. https://doi.org/10.1128/jb.172.6 .3073-3080.1990.

50. Broomfield RJ, Morgan SD, Khan A, Stickler DJ. 2009. Crystalline bacterial biofilm formation on urinary catheters by urease-producing urinary tract pathogens: a simple method of control. J Med Microbiol 58:1367-1375. https://doi.org/10.1099/jmm.0.012419-0.

51. Jones GL, Muller CT, O'Reilly M, Stickler DJ. 2006. Effect of triclosan on the development of bacterial biofilms by urinary tract pathogens on urinary catheters. J Antimicrob Chemother 57:266-272. https://doi.org/ 10.1093/jac/dki447.
52. Jones BD, Mobley HL. 1987. Genetic and biochemical diversity of ureases of Proteus, Providencia, and Morganella species isolated from urinary tract infection. Infect Immun 55:2198-2203.

53. Liu H, Zhu J, Hu Q, Rao X. 2016. Morganella morganii, a non-negligent opportunistic pathogen. Int J Infect Dis 50:10-17. https://doi.org/10 .1016/j.ijid.2016.07.006.

54. Erlanger D, Assous MV, Wiener-Well Y, Yinnon AM, Ben-Chetrit E. 2017. Clinical manifestations, risk factors, and prognosis of patients with Morganella morganii sepsis. J Microbiol Immunol Infect 52:443-448. https:// doi.org/10.1016/j.jmii.2017.08.010.

55. Laupland KB, Parkins MD, Ross T, Pitout JD. 2007. Population-based laboratory surveillance for tribe Proteeae isolates in a large Canadian health region. Clin Microbiol Infect 13:683-688. https://doi.org/10.1111/ j.1469-0691.2007.01715.x.

56. Stickler D, Ganderton L, King J, Nettleton J, Winters C. 1993. Proteus mirabilis biofilms and the encrustation of urethral catheters. Urol Res 21:407-411. https://doi.org/10.1007/bf00300077.

57. Armbruster C, Mobley H, Pearson M. 8 February 2018, posting date. Pathogenesis of Proteus mirabilis infection. EcoSal Plus 2018 https://doi .org/10.1128/ecosalplus.ESP-0009-2017.

58. Stickler DJ, Lear JC, Morris NS, Macleod SM, Downer A, Cadd DH, Feast WJ. 2006. Observations on the adherence of Proteus mirabilis onto polymer surfaces. J Appl Microbiol 100:1028-1033. https://doi.org/10 .1111/j.1365-2672.2006.02840.x.

59. Flores-Mireles AL, Walker JN, Caparon M, Hultgren SJ. 2015. Urinary tract infections: epidemiology, mechanisms of infection, and treatment options. Nat Rev Microbiol 13:269-284. https://doi.org/10.1038/nrmicro3432.

60. Tien BYQ, Goh HMS, Chong KKL, Bhaduri-Tagore S, Holec S, Dress R, Ginhoux F, Ingersoll MA, Williams RBH, Kline KA. 2017. Enterococcus faecalis promotes innate immune suppression and polymicrobial catheter-associated urinary tract infection. Infect Immun 85:e00378-17. https://doi.org/10.1128/IAI.00378-17.

61. Kline KA, Schwartz DJ, Gilbert NM, Hultgren SJ, Lewis AL. 2012. Immune modulation by group B Streptococcus influences host susceptibility to urinary tract infection by uropathogenic Escherichia coli. Infect Immun 80:4186-4194. https://doi.org/10.1128/IAI.00684-12.

62. Alteri CJ, HimpsI SD, Mobley HL. 2015. Preferential use of central metabolism in vivo reveals a nutritional basis for polymicrobial infection. PLoS Pathog 11:e1004601. https://doi.org/10.1371/journal.ppat.1004601.

63. Hjelm E, Lundell-Etherden I, Mårdh PA. 1987. Ascending urinary tract infections in rats induced by Staphylococcus saprophyticus and Proteus mirabilis. Acta Pathol Microbiol Immunol Scand B 95:347-350.

64. Tsuchimori N, Hayashi R, Shino A, Yamazaki T, Okonogi K. 1994. Enterococcus faecalis aggravates pyelonephritis caused by Pseudomonas aeruginosa in experimental ascending mixed urinary tract infection in mice. Infect Immun 62:4534-4541.

65. Ohlemacher SI, Giblin DE, d'Avignon DA, Stapleton AE, Trautner BW, Henderson JP. 2017. Enterobacteria secrete an inhibitor of Pseudomonas virulence during clinical bacteriuria. J Clin Invest 127:4018-4030. https://doi.org/10.1172/JCI92464.

66. Storm DW, Koff SA, Horvath DJ, Jr, Li B, Justice SS. 2011. In vitro analysis of the bactericidal activity of Escherichia coli Nissle 1917 against pediatric uropathogens. J Urol 186:1678-1683. https://doi.org/10.1016/j.juro.2011 .04 .021 .

67. Darouiche RO, Riosa S, Hull RA. 2010. Comparison of Escherichia coli strains as agents for bacterial interference. Infect Control Hosp Epidemiol 31:659-661. https://doi.org/10.1086/653070.

68. Mobley HL, Green DM, Trifillis AL, Johnson DE, Chippendale GR, Lockatell CV, Jones BD, Warren JW. 1990. Pyelonephritogenic Escherichia coli and killing of cultured human renal proximal tubular epithelial cells: role of hemolysin in some strains. Infect Immun 58:1281-1289.

69. Burall LS, Harro JM, Li X, Lockatell CV, HimpsI SD, Hebel JR, Johnson DE, Mobley H. 2004. Proteus mirabilis genes that contribute to pathogenesis of urinary tract infection: identification of 25 signature-tagged mutants attenuated at least 100-fold. Infect Immun 72:2922-2938. https://doi .org/10.1128/iai.72.5.2922-2938.2004.

70. Island MD, Mobley HL. 1995. Proteus mirabilis urease: operon fusion and linker insertion analysis of ure gene organization, regulation, and function. J Bacteriol 177:5653-5660. https://doi.org/10.1128/jb.177.19.5653 $-5660.1995$.

71. Alamuri P, Eaton KA, HimpsI SD, Smith SN, Mobley H. 2009. Vaccination with proteus toxic agglutinin, a hemolysin-independent cytotoxin in vivo, protects against Proteus mirabilis urinary tract infection. Infect Immun 77:632-641. https://doi.org/10.1128/IAI.01050-08. 
72. Guiton PS, Hung CS, Hancock LE, Caparon MG, Hultgren SJ. 2010. Enterococcal biofilm formation and virulence in an optimized murine model of foreign body-associated urinary tract infections. Infect Immun 78:4166-4175. https://doi.org/10.1128/IAI.00711-10.

73. Kadurugamuwa JL, Modi K, Yu J, Francis KP, Purchio T, Contag PR. 2005 Noninvasive biophotonic imaging for monitoring of catheter-associated urinary tract infections and therapy in mice. Infect Immun 73 3878-3887. https://doi.org/10.1128/IAI.73.7.3878-3887.2005.

74. Kurosaka Y, Ishida Y, Yamamura E, Takase H, Otani T, Kumon H. 2001. A non-surgical rat model of foreign body-associated urinary tract infection with Pseudomonas aeruginosa. Microbiol Immunol 45:9-15. https://doi .org/10.1111/j.1348-0421.2001.tb01268.x.

75. Lloyd AL, Smith SN, Eaton KA, Mobley HL. 2009. Uropathogenic Escherichia coli suppresses the host inflammatory response via pathogenicity island genes sisA and sisB. Infect Immun 77:5322-5333. https://doi.org/ 10.1128/IAI.00779-09.
76. Eaton KA, Friedman DI, Francis GJ, Tyler JS, Young VB, Haeger J, Abu-Ali G, Whittam TS. 2008. Pathogenesis of renal disease due to enterohemorrhagic Escherichia coli in germ-free mice. Infect Immun 76:3054-3063. https://doi.org/10.1128/IAI.01626-07.

77. Goswami K, Chen C, Xiaoli L, Eaton KA, Dudley EG. 2015. Coculture of Escherichia coli 0157:H7 with a nonpathogenic E. coli strain increases toxin production and virulence in a germfree mouse model. Infect Immun 83:4185-4193. https://doi.org/10.1128/IAI.00663-15.

78. Neoh KG, Li M, Kang E-T, Chiong E, Tambyah PA. 2017. Surface modification strategies for combating catheter-related complications: recent advances and challenges. J Mater Chem B 5:2045-2067. https://doi.org/ 10.1039/C6TB03280J.

79. Siddiq DM, Darouiche RO. 2012. New strategies to prevent catheterassociated urinary tract infections. Nat Rev Urol 9:305-314. https://doi .org/10.1038/nrurol.2012.68. 